\title{
La atención y prevención de la violencia en contra de la mujer en Guatemala: Directorio de servicios
}

Diana Del Castillo

Elvia Raquec

Follow this and additional works at: https://knowledgecommons.popcouncil.org/departments_sbsr-pgy

Part of the Domestic and Intimate Partner Violence Commons, Family, Life Course, and Society Commons, and the International Public Health Commons How does access to this work benefit you? Let us know!

\section{Recommended Citation}

Del Castillo, Diana and Elvia Raquec. 2011. "La atención y prevención de la violencia en contra de la mujer en Guatemala: Directorio de servicios." New York: Population Council. 


\section{LA ATENCIÓN Y PREVENCIÓN DE LA VIOLENCIA EN CONTRA DE LA MUJER EN GUATEMALA}

\section{DIRECTORIO DE SERVICIOS}

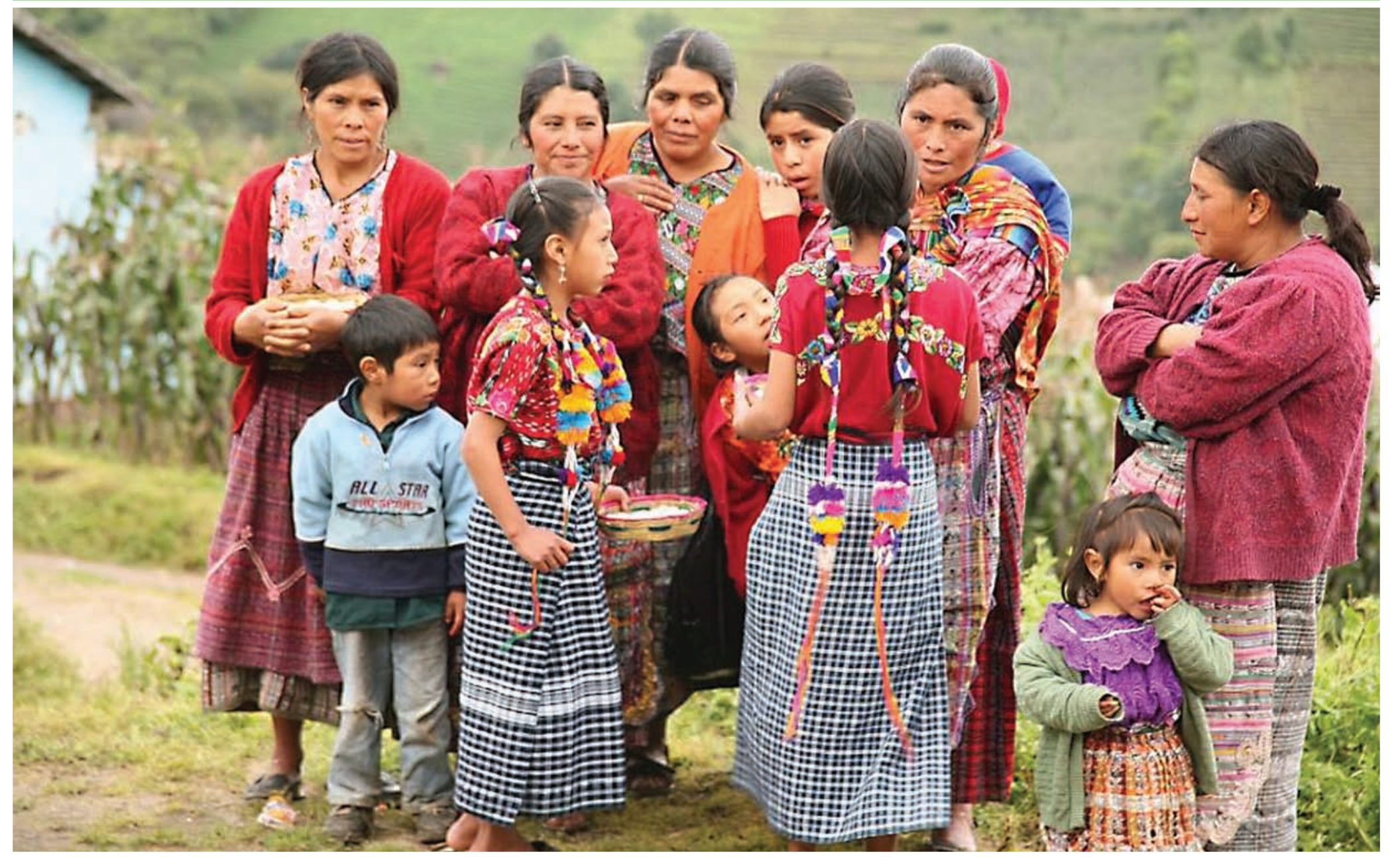

Diana Del Castillo Elvia Raquec 
(c) 2011 The Population Council, Inc.

\section{(2) Population Council}

El Population Council realiza investigación en todo el mundo con el fin de mejorar políticas, programas y productos en tres áreas: VIH y SIDA; pobreza, género y juventud; y salud reproductiva.

Population Council

One Dag Hammarskjold Plaza

New York, NY 10017 USA

www.popcouncil.org

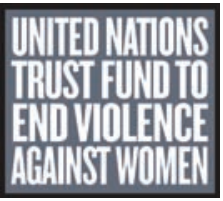

Este Fondo Fiduciario fue creado en el año 1996 por la Asamblea General de las Naciones Unidas, para "brindar, a nivel comunitario, regional e internacional, la asistencia técnica necesaria para impulsar el desarrollo de modelos innovadores e intervenciones estratégicas en el área de violencia contra las mujeres."

\section{Autoras \\ Diana Del Castillo \\ Elvia Raquec}

\section{Contribuciones}

Alejandra Colom

Jennifer Catino

Foto de portada: Fernando Velásquez

Diseño: Mike Vosika

El contenido de esta publicación puede ser parcial o totalmente reproducido sin el permiso de las autoras o la editorial, siempre y cuando todos los derechos de autor sean debidamente reconocidos y las copias sean distribuidas sin ningún costo. Cualquier reproducción con fines comerciales deberá obtener el previo permiso por escrito del Population Council. 


\section{ÍNDICE}

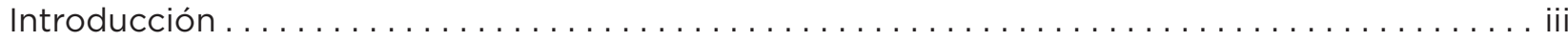

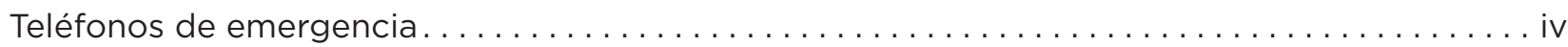

\section{SERVICIOS DE ATENCIÓN}

Alta Verapaz

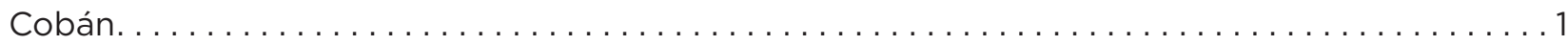

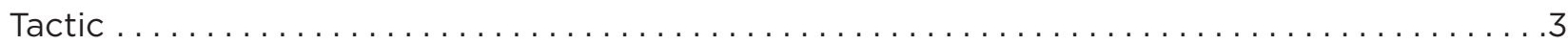

Chimaltenango

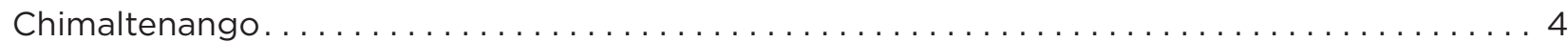

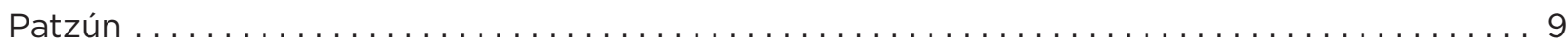

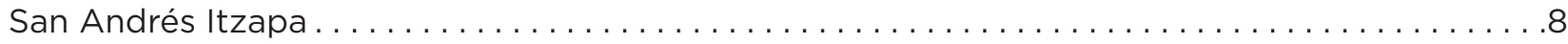

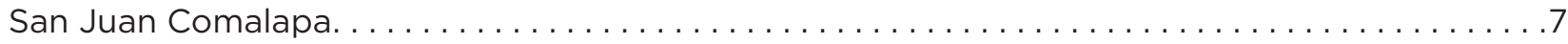

Guatemala

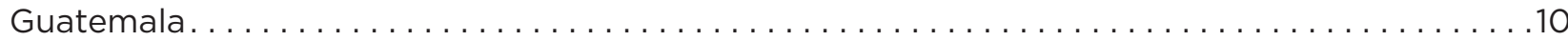

Quetzaltenango

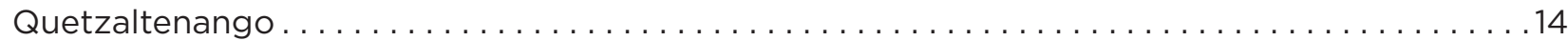

Sacatepéquez

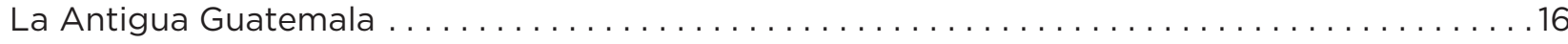

Sololá

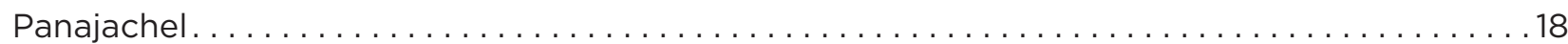

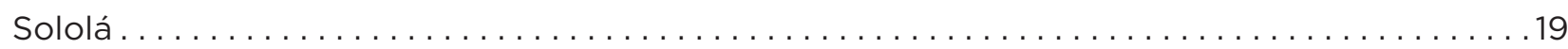

Totonicapán

Totonicapán ................................................. 20

OTROS SERVICIOS Y RECURSOS

Alta Verapaz

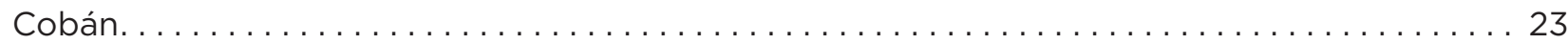

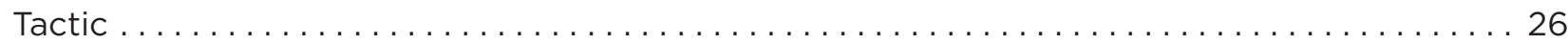

Chimaltenango

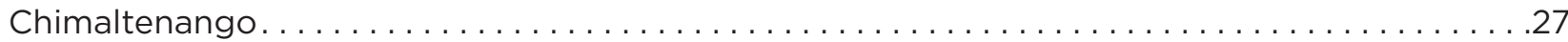

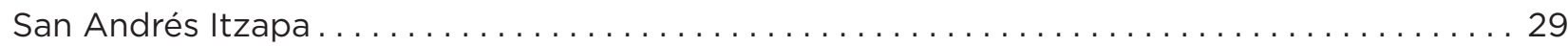

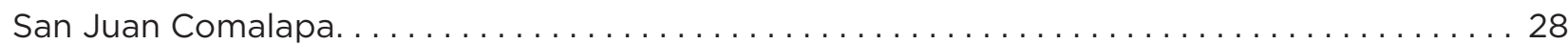

Guatemala

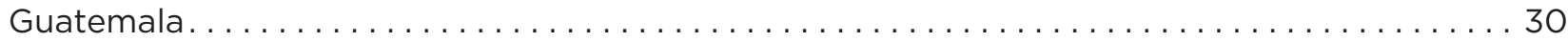

Quetzaltenango

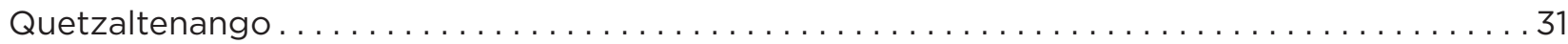

Sacatepéquez

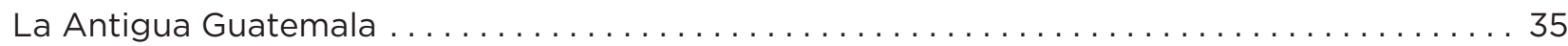

Sololá

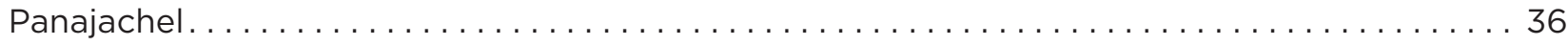

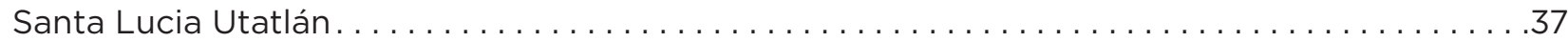

Santiago Atitlán. . . . . . . . . . . . . . . . . . . . . . . . . . . . . . . . . 39

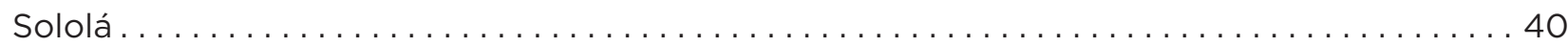

Totonicapán

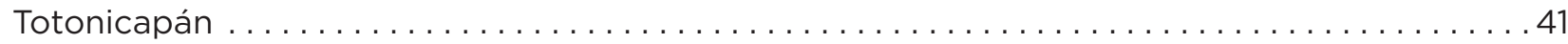

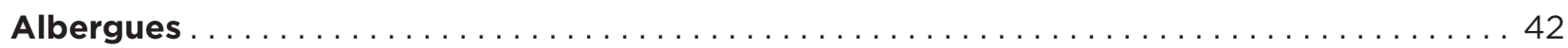





\section{INTRODUCCIÓN}

La violencia en contra de la mujer se ha definido como "Todo acto de violencia basado en la pertenencia al sexo femenino que tenga o pueda tener como resultado un daño o sufrimiento físico, sexual o psicológico para la mujer, así como las amenazas de tales actos, la coacción o la privación arbitraria de la libertad, tanto si se producen en la vida pública como en la vida privada" (ESC 1992). La violencia contra la mujer es un grave problema de salud pública, constituye una violación de los derechos humanos y es una manifestación de extrema inequidad de género dentro de una sociedad. Las consecuencias son de corto y largo plazo, y pueden afectar la salud física, mental, sexual y reproductiva de la mujer, comprometiendo el sano desarrollo de los hijos, así como la seguridad y estabilidad de la familia (WHO 2011).

El problema de violencia en contra de la mujer en Guatemala es uno de los más severos en Latinoamérica (UNDP 2011). La mayoría de las víctimas son mujeres pobres de áreas rurales, jóvenes, con edades entre los 13 y 30 años (GHRC 2007).

El Directorio de proveedores de servicios y recursos para la atención y prevención de la violencia en contra de las niñas, adolescentes y mujeres se realizó como parte del programa del Population Council "Abriendo Oportunidades". Este programa tiene como uno de sus objetivos la prevención de la violencia de género en Guatemala a través de la creación de espacios seguros en los cuales se promuevan actitudes y comportamientos libres de violencia a nivel comunitario, donde niñas y jóvenes indígenas de áreas rurales pueden reunirse y aprender sobre temas y habilidades para la vida, ampliar sus redes de amigas y formarse como líderes y agentes de cambio social dentro de su comunidad.

A través de las actividades de prevención de violencia de género de "Abriendo Oportunidades" se ha detectado un número cada vez mayor de niñas y jóvenes indígenas, especialmente de áreas rurales, que han sido afectadas o están en riesgo de sufrir cualquiera de las manifestaciones de este tipo de violencia. Dentro del marco de la implementación del proyecto
"Volviendo seguro el entorno en comunidades indígenas de Guatemala: la prevención primaria de la violencia de género a través de la formación y mentoría de jóvenes mayas agentes de cambio social," apoyado por el Fondo Fiduciario de la ONU para la Eliminación de la Violencia contra las Mujeres, surgió la urgente necesidad de conocer recursos y servicios sobre violencia en contra de la mujer, apropiados y confiables, a donde se puedan referir las niñas, adolescentes y mujeres que los requieran.

Este directorio es el resultado del mapeo institucional realizado en siete departamentos de la República de Guatemala, que incluye Guatemala, Sacatepéquez, Chimaltenango, Sololá, Alta Verapaz, Quetzaltenango y Totonicapán, lugares donde opera el programa Abriendo Oportunidades y donde se identificaron proveedores de servicios tanto de atención como de prevención de violencia basada en género, accesibles para mujeres, jóvenes y niñas.

Adicionalmente, a través de este proyecto el Population Council pretende lograr la máxima difusión de la información obtenida a través del mapeo institucional entre todas las organizaciones que prestan servicios relacionados con la violencia en contra de la mujer. El objetivo es promover el conocimiento acerca de los servicios y recursos disponibles, favorecer la comunicación interinstitucional y facilitar la cooperación entre los diferentes entes gubernamentales y no gubernamentales trabajando en el área para contar con servicios de calidad, pertinentes culturalmente y adaptados a las necesidades particulares de niñas y adolescentes.

\section{Referencias}

Economic and Social Council (ESC). 1992. "Report of the Working Group of Violence against Women." E/CN.6WG.2/1992/11.3. Viena: Naciones Unidas.

Guatemala Human Rights Commission (GHRC). 2007. Three Thousand and Counting: A Report on Violence against Women in Guatemala. Descargado de: <www.ghrc-usa.org>.

United Nations Development Programme (UNDP). 2011. Human Development Reports. Descargado de: <http://hdr.undp.org/en/statistics/>.

World Health Organization (WHO). 2010. "Violence against Women.” Descargado de: <http://www.who. int/mediacentre/factsheets/fs239/en/index.html>. 


\section{TELÉFONOS DE EMERGENCIA 24 HORAS}

Nuevos Horizontes: 52410801 / 54226572 / 53815930

Médicos Sin Fronteras (Violencia Sexual): 51938281 / 24745830

Grupo Guatemalteco de Mujeres (GGM): 53086448

CAIMU: 23890500

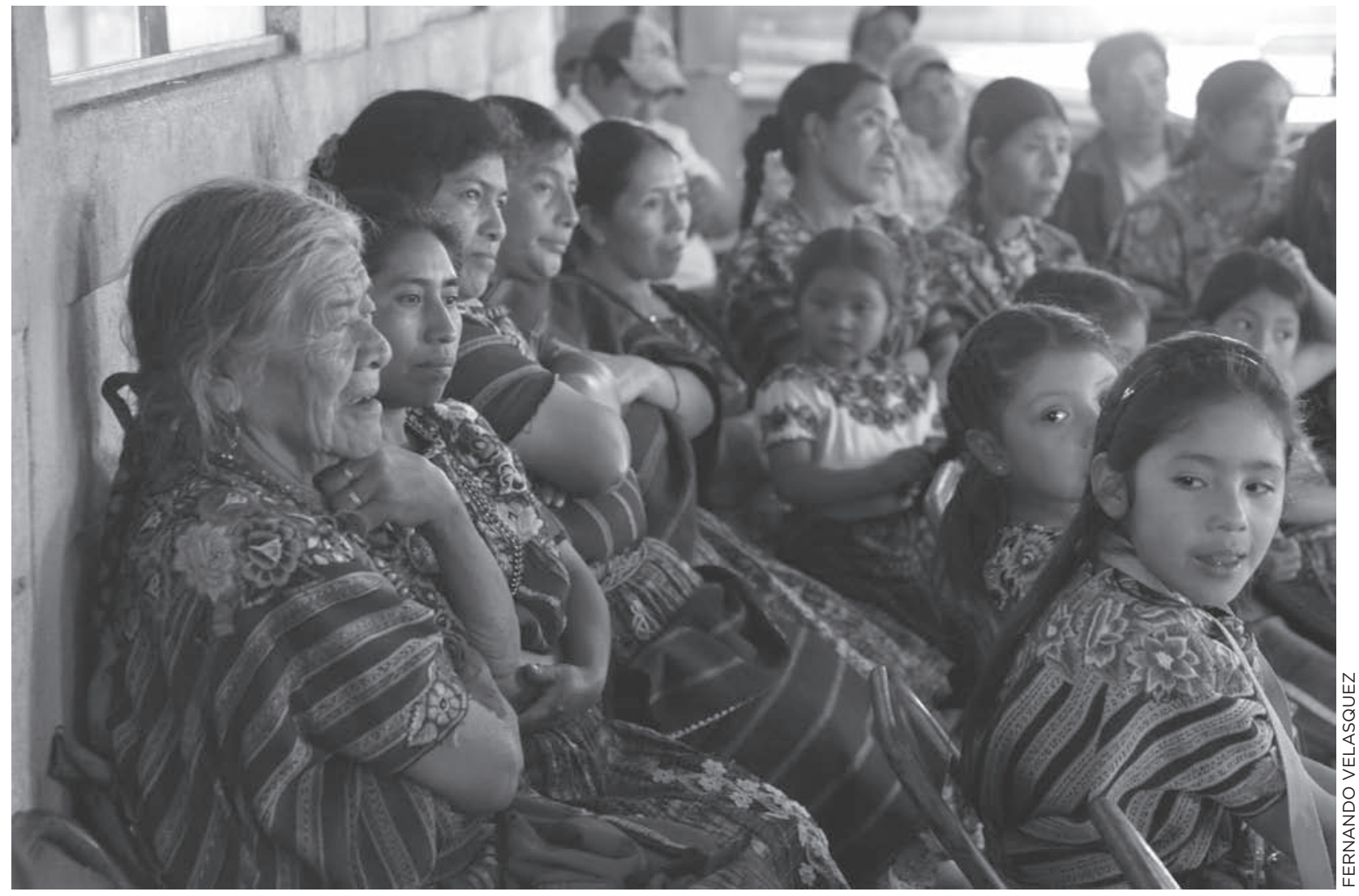




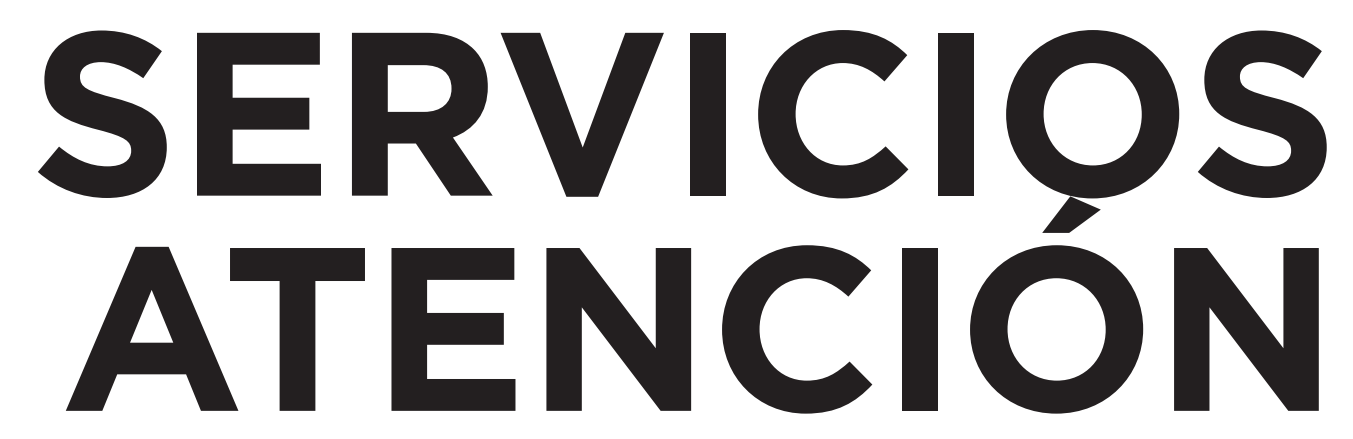





\section{ALTA VERAPAZ}

\section{Cobán}

\begin{tabular}{|l|l|}
\hline Nombre institucional & $\begin{array}{l}\text { Centro de Apoyo Integral para Mujeres Sobrevivientes de la } \\
\text { Violencia Intrafamiliar - Ak'Yu'Am }\end{array}$ \\
\hline Misión & $\begin{array}{l}\text { Apoyo integral a mujeres sobrevivientes de violencia intrafamiliar. } \\
\text { Brindar atención personalizada y multidisciplinaria, promoviendo la } \\
\text { prevención y erradicación de la violencia en Alta Verapaz y el respeto a } \\
\text { los derechos de las mujeres. }\end{array}$ \\
\hline Servicios & $\begin{array}{l}\text { Atención legal, psicológica y social. Albergue para mujeres mayores de } \\
\text { edad e hijos pequeños, cuentan con enfermera. }\end{array}$ \\
\hline Dirección & $\begin{array}{l}\text { Una cuadra antes Colegio Verapaz, Cobán, Alta Verapaz (esquina de la } \\
\text { Departamental de Educación) }\end{array}$ \\
\hline Teléfono & 4025-4353; fax 7952-2687; cel 5780-1201 \\
\hline Correo electrónico & akyuam.cejav@yahoo.es; cejcav@yahoo.es \\
\hline Cobertura & Comunidades y municipios de Alta Verapaz \\
\hline Contacto & Vicky Mo y Lorni Marcela Bac, Trabajadora Social y Oficinista \\
\hline
\end{tabular}

\begin{tabular}{|l|l|}
\hline Nombre institucional & DEMI - Defensoría de la Mujer Indígena \\
\hline Misión & $\begin{array}{l}\text { Defender y promover el pleno ejercicio de los derechos de las mujeres } \\
\text { indígenas para contribuir a la erradicación de todas las formas de } \\
\text { violencia y discriminación contra la mujer indígena. }\end{array}$ \\
\hline Servicios & $\begin{array}{l}\text { Atención en idioma indígena y con pertinencia cultural. Asistencia ju- } \\
\text { rídica, social y psicológica. Procesos pensión alimenticia. }\end{array}$ \\
\hline Dirección & Municipalidad Cobán, Alta Verapaz \\
\hline Teléfono & 7952-9866 \\
\hline Correo electrónico & demicoban@yahoo.com \\
\hline Cobertura & Todo los municipios de Alta Verapaz \\
\hline Contacto & Licda. Miriam Mus, Directora Área Social \\
\hline
\end{tabular}




\section{Cobán}

\begin{tabular}{|l|l|}
\hline Nombre institucional & Área de Salud - Programa Prevención de Violencia Sexual \\
\hline Misión & $\begin{array}{l}\text { Coordinadora interinstitucional para la prevención de la violencia } \\
\text { sexual en Alta Verapaz. }\end{array}$ \\
\hline Servicios & $\begin{array}{l}\text { Unidad de salud mental. Orientación y servicios directos a víctimas de } \\
\text { violencia sexual. Mesa Departamental sobre la violencia. }\end{array}$ \\
\hline Dirección & 7 calle 6-24 zona 11, Cobán Alta Verapaz \\
\hline Teléfono & $7952-1189$ \\
\hline Correo electrónico & saludmentaldasav@gmail.com \\
\hline Cobertura & Alta Verapaz \\
\hline Contacto & Licda. Ulia Zugeidy Figueroa García, Psicóloga \\
\hline
\end{tabular}

\begin{tabular}{|c|c|}
\hline Nombre institucional & Refugio de la Niñez \\
\hline Misión & Atención de casos niños y niñas víctimas de violencia sexual y tráfico. \\
\hline Servicios & $\begin{array}{l}\text { Atención psicológica, investigación social, apoyo jurídico y seguimiento } \\
\text { de casos de niños y niñas víctimas de violencia sexual. Prevención a } \\
\text { través de promotoras con líderes, estudiantes, comunidades, mujeres } \\
\text { y padres de familia. Procesos psicoterapéuticos una vez al mes con } \\
\text { padres y de acuerdo a las necesidades. Programa de Familia Sustituta } \\
\text { apoyado por UNICEF. }\end{array}$ \\
\hline Dirección & $\begin{array}{l}7 \text { Av. 2-29 "A" zona } 1 \text { Calle del Calvario, Cobán Alta Verapaz; } 11 \text { avenida } \\
\text { 16-40 zona 1, Ciudad de Guatemala }\end{array}$ \\
\hline Teléfono & 7992-3539, (502) 2253-7976 \\
\hline Correo electrónico & www.refugiodelaninez.org.gt \\
\hline Cobertura & Alta y Baja Verapaz, El Progreso \\
\hline Contacto & $\begin{array}{l}\text { Eliria Floridalma Pop, Trabajadora Social; Balbina Sierra Carrera, } \\
\text { Coordinadora }\end{array}$ \\
\hline
\end{tabular}




\section{Tactic}

\begin{tabular}{|l|l|}
\hline Nombre institucional & Centro de Salud \\
\hline Misión & Servicios médicos. \\
\hline Servicios & $\begin{array}{l}\text { Atención médica inmediata a mujeres y niñas víctimas de violencia. } \\
\text { Siguen el protocolo violencia sexual y kit de emergencia. Servicios de } \\
\text { psicología. Remiten casos a Cobán. }\end{array}$ \\
\hline Dirección & Tactic, Alta Verapaz \\
\hline Teléfono & $7953-9191$ \\
\hline Correo electrónico & No disponible \\
\hline Cobertura & Alta Verapaz \\
\hline Contacto & Marlin Corina Aldana Espinosa, Médica General \\
\hline
\end{tabular}

\begin{tabular}{|l|l|}
\hline Nombre institucional & Juzgado de Paz \\
\hline Misión & $\begin{array}{l}\text { Delitos y hechos de violencia de todo tipo. Llevar a cabo denuncias y } \\
\text { procesos legales. }\end{array}$ \\
\hline Servicios & Servicios legales a mujeres víctimas de violencia. \\
\hline Dirección & 8a. Avenida, 4-12, Zona 5, Barrio la Asunción Tactic \\
\hline Teléfono & $7953-9174$ / cel. 5185-2940 \\
\hline Correo electrónico & No disponible \\
\hline Cobertura & Alta Verapaz \\
\hline Contacto & Licda. Patricia Acjam Leal, Jueza \\
\hline
\end{tabular}




\section{CHIMALTENANGO}

\section{Chimaltenango}

\begin{tabular}{|l|l|}
\hline Nombre institucional & $\begin{array}{l}\text { CICAM - Centro de Investigación, Capacitación y Apoyo a la } \\
\text { Mujer }\end{array}$ \\
\hline Misión & $\begin{array}{l}\text { Servicio, asistencia y desarrollo integral de la mujer, que especial- } \\
\text { mente persigue el estudio, la investigación, capacitación y apoyo a } \\
\text { la mujer Guatemalteca. }\end{array}$ \\
\hline Servicios & $\begin{array}{l}\text { Apoyo legal y orientación psicológica a mujeres víctimas de violencia. } \\
\text { Asesoría a organizaciones que se interesen en el tema de los derechos } \\
\text { de las mujeres y la prestación de servicios a favor de ellas. Atención } \\
\text { en crisis, evaluación psicológica, grupos de autoayuda y terapias de } \\
\text { seguimiento. Terapia ocupacional. }\end{array}$ \\
\hline Dirección & 2da. Ave. Lote No. 94 Zona 1, Chimaltenango \\
\hline Teléfono & 7849-1628 / 4271-7878 \\
\hline Correo electrónico & cicam.chimaltenango09@gmail.com; www.cicam.org \\
\hline Cobertura & Todo el departamento \\
\hline Contacto & Licda. Lucia Fernanda Quiñonez Pérez, Abogada y Notaria \\
\hline
\end{tabular}

\begin{tabular}{|l|l|}
\hline Nombre institucional & Generando \\
\hline Misión & $\begin{array}{l}\text { Generar y facilitar oportunidades de empoderamiento e } \\
\text { independencia económica para mujeres jóvenes como forma para } \\
\text { combatir la violencia, embarazos en adolescentes y la falta de } \\
\text { oportunidades económicas en el área de Chimaltenango. }\end{array}$ \\
\hline Servicios & $\begin{array}{l}\text { Crear un centro de atención integral para mujeres sobrevivientes de } \\
\text { violencia. Atención psicológica y asesoria legal. }\end{array}$ \\
\hline Dirección & 1. Av 3-39 Zona 3, Chimaltenango \\
\hline Teléfono & 7849-4360 \\
\hline Correo electrónico & asoc.generando@gmail.com; www.associationgenerando.org \\
\hline Cobertura & Todo el municipio de Chimaltenango \\
\hline Contacto & $\begin{array}{l}\text { Danessa Luna, Directora Ejecutiva; Cristina Chiquin, Programa de } \\
\text { Incidencia Política; Nayeli Lopez, Procuradora }\end{array}$ \\
\hline
\end{tabular}




\section{Chimaltenango}

\begin{tabular}{|l|l|}
\hline Nombre institucional & Hospital Nacional \\
\hline Misión & $\begin{array}{l}\text { Brindar servicio de psicología a mujeres sobrevivientes de cualquier } \\
\text { tipo de violencia. }\end{array}$ \\
\hline Servicios & Servicios de psicología para todo tipo de pacientes. \\
\hline Dirección & La Alameda, Chimaltenango. \\
\hline Teléfono & 7839-1504, ext. 509 \\
\hline Correo electrónico & No disponible \\
\hline Cobertura & Municipio y departamento de Chimaltenango \\
\hline Contacto & $\begin{array}{l}\text { Licdo. Otto Byron Chacón Zarazua, Psicólogo; Mary Leonora Choy, } \\
\text { Asistente }\end{array}$ \\
\hline
\end{tabular}

\begin{tabular}{|l|l|}
\hline Nombre institucional & Instituto de la Defensa Pública \\
\hline Misión & $\begin{array}{l}\text { A raíz de la Ley de Violencia Intrafamiliar se creó una Unidad } \\
\text { de Asistencia Legal Gratuita a Mujeres maltratadas, a quienes se atien- } \\
\text { den inmediatamente. }\end{array}$ \\
\hline Servicios & $\begin{array}{l}\text { Servicios legales y asesoría jurídica gratuitos. Estudios socio- } \\
\text { económicos, visitas domiciliarias. Unidad intercultural. }\end{array}$ \\
\hline Dirección & 2da Calle 8-177 Zona 3, Chimaltenango \\
\hline Teléfono & 7839-5390 / 7879-9186 \\
\hline Correo electrónico & No disponible \\
\hline Cobertura & Todo el departamento de Chimaltenango \\
\hline Contacto & $\begin{array}{l}\text { Licdo. Mario Humberto Smith Angel, Director; } \\
\text { Licda. Gladys Aracely Rivera Estrada, Trabajadora Social }\end{array}$ \\
\hline
\end{tabular}




\section{Chimaltenango}

\begin{tabular}{|l|l|}
\hline Nombre institucional & PNC Delegación de Equidad de Género \\
\hline Misión & $\begin{array}{l}\text { Atención de casos de trata de personas, femicidios, violencia sexual } \\
\text { e intrafamiliar. }\end{array}$ \\
\hline Servicios & $\begin{array}{l}\text { Prevención y atención de casos sobre violencia en contra de las } \\
\text { mujeres en comunidades rurales del departamento. }\end{array}$ \\
\hline Dirección & Colonia Santa Teresita, Chimaltenango \\
\hline Teléfono & 4055-9398 \\
\hline Correo electrónico & No disponible \\
\hline Cobertura & Chimaltenango \\
\hline Contacto & $\begin{array}{l}\text { Licda. Dora Mejía González, Dirección General Equidad de Género, } \\
\text { Guatemala }\end{array}$ \\
\hline
\end{tabular}

\begin{tabular}{|l|l|}
\hline Nombre institucional & Presbiterio Kaqchikel, Proyecto Tabita Levántate \\
\hline Misión & $\begin{array}{l}\text { Proyecto dirigido especialmente a las mujeres maltratadas, humilla- } \\
\text { das, golpeadas, discriminadas de Chimaltenango para ayudarles a } \\
\text { sanar tanto en su interior como en su exterior y que valoren a si } \\
\text { mismas y que encuentren amor y sentido en la vida. }\end{array}$ \\
\hline Servicios & $\begin{array}{l}\text { Oficina de atención integral a la mujer incluye servicios legales, } \\
\text { psicológicos, y médicos para mujeres víctimas de cualquier tipo de } \\
\text { violencia. Además de ayuda y orientación espiritual. }\end{array}$ \\
\hline Dirección & 2da Calle 8-72 Zona 3, Chimaltenango \\
\hline Teléfono & 7839-1503 \\
\hline Correo electrónico & www.presbiteriokaqchikel.org, info@presbiteriokaqchikel \\
\hline Cobertura & Nivel departamental \\
\hline Contacto & Carolina Alvarado, Abogada y Notaria; Magda Argueta, Psicóloga \\
\hline
\end{tabular}


Patzún

\begin{tabular}{|l|l|}
\hline Nombre institucional & Juzgado de Paz \\
\hline Misión & Prestar asesoría legal y procesar denuncias. \\
\hline Servicios & Solo área jurídica. \\
\hline Dirección & $5^{a}$ calle 2-38, zona 1, Patzún Chimaltenango \\
\hline Teléfono & $7839-8301$ \\
\hline Correo electrónico & No disponible \\
\hline Cobertura & Chimaltenango \\
\hline Contacto & Licda. Lilian Valenzuela, Jueza \\
\hline
\end{tabular}




\section{San Andrés Itzapa}

\begin{tabular}{|l|l|}
\hline Nombre institucional & Oficina Municipal de la Mujer \\
\hline Misión & $\begin{array}{l}\text { Apoyo a } 35 \text { grupos de mujeres organizadas a través de la } \\
\text { municipalidad. }\end{array}$ \\
\hline Servicios & $\begin{array}{l}\text { Apoyo y acompañamiento a mujeres que sufren violencia. Ayudas con } \\
\text { medicamentos y jornadas de exámenes médicos. }\end{array}$ \\
\hline Dirección & Edificio Municipal Itzapa, Chimaltenango \\
\hline Teléfono & 7849-4601 / 4630-4135 \\
\hline Correo electrónico & glaynezchavac@yahoo.es \\
\hline Cobertura & Municipio \\
\hline Contacto & Gloria Laynez, Coordinadora \\
\hline
\end{tabular}




\section{San Juan Comalapa}

\begin{tabular}{|l|l|}
\hline Nombre institucional & Juzgado de Paz \\
\hline Misión & Procesos legales a víctimas de violencia y otros delitos. \\
\hline Servicios & $\begin{array}{l}\text { Recibir y procesar denuncias de violencia en contra de la mujer y } \\
\text { todo tipo. }\end{array}$ \\
\hline Dirección & O Calle 3-69 Zona 1 \\
\hline Teléfono & $7849-8892$ \\
\hline Correo electrónico & No disponible \\
\hline Cobertura & Municipio \\
\hline Contacto & Rosa Vidal, Oficial III \\
\hline
\end{tabular}

\begin{tabular}{|l|l|}
\hline Nombre institucional & Oficina Municipal de la Mujer \\
\hline Misión & $\begin{array}{l}\text { Promover programas de desarrollo productivo. Acompañamiento a } \\
\text { trámites y citas legales de mujeres víctimas de violencia. }\end{array}$ \\
\hline Servicios & $\begin{array}{l}\text { Dar apoyo para el desarrollo económico de la mujer. Acompañamiento } \\
\text { y orientación legal para denuncias. }\end{array}$ \\
\hline Dirección & Municipalidad, Comalapa \\
\hline Teléfono & 5628-8203 / 5787-3450 \\
\hline Correo electrónico & oficina.chitox@gmail.com \\
\hline Cobertura & Área rural y urbana Comalapa \\
\hline Contacto & Glendy Tubin, Secretaria \\
\hline
\end{tabular}




\section{GUATEMALA}

Guatemala

\begin{tabular}{|l|l|}
\hline Nombre institucional & APROFAM \\
\hline Misión & $\begin{array}{l}\text { Programa salud sexual y reproductiva para adolescentes y } \\
\text { sobrevivientes de violencia sexual. }\end{array}$ \\
\hline Servicios & $\begin{array}{l}\text { Brindar atención médica inmediata. Orientación sobre consecuencias } \\
\text { y alternativas en casos de violencia sexual. Charlas en establecimientos } \\
\text { públicos y privados sobre salud sexual, autoestima, paternidad } \\
\text { responsable, noviazgo, etc. }\end{array}$ \\
\hline Dirección & 1ra Av 8-36 zona 1, Guatemala \\
\hline Teléfono & 4402-5438 \\
\hline Correo electrónico & vbg.adolescentes@aprofam.org.gt \\
\hline Cobertura & Guatemala \\
\hline Contacto & $\begin{array}{l}\text { Licda. Lily Rivera Pineda, Coordinadora del Programa Adolescentes. } \\
\text { Licda. María Julia Alcántara }\end{array}$ \\
\hline
\end{tabular}

\begin{tabular}{|l|l|}
\hline Nombre institucional & $\begin{array}{l}\text { CICAM - Centro de Investigación, Capacitación y Apoyo a la } \\
\text { Mujer }\end{array}$ \\
\hline Misión & $\begin{array}{l}\text { Servicio, asistencia y desarrollo integral de la mujer, que especialmente } \\
\text { persigue el estudio, la investigación, capacitación y apoyo a la mujer } \\
\text { guatemalteca. }\end{array}$ \\
\hline Servicios & $\begin{array}{l}\text { Apoyo legal y orientación psicológica a mujeres víctimas de violencia. } \\
\text { Asesoría a organizaciones que se interesen en el tema de los derechos } \\
\text { de las mujeres y la prestación de servicios a favor de ellas. Atención } \\
\text { en crisis, evaluación psicológica, grupos de autoayuda y terapias de } \\
\text { seguimiento. Terapia ocupacional. }\end{array}$ \\
\hline Dirección & 6 Av 0-60 zona 4 Torre Profesional 1, Nivel 2, Of. 203, Guatemala \\
\hline Teléfono & 2335-1779 / 2335-2165 \\
\hline Correo electrónico & cicam@cicam.org.gt \\
\hline Cobertura & Ciudad de Guatemala \\
\hline Contacto & Angélica Valenzuela, Coordinadora \\
\hline
\end{tabular}




\section{Guatemala}

\begin{tabular}{|l|l|}
\hline Nombre institucional & CONACMI - Comisión Nacional Contra el Maltrato Infantil \\
\hline Misión & $\begin{array}{l}\text { Asociación civil encargada de coordinar acciones con entes del } \\
\text { gobierno y no gubernamentales, orientadas a la prevención y atención } \\
\text { del maltrato y abuso sexual infantil y otros abusos contra la juventud. }\end{array}$ \\
\hline Servicios & $\begin{array}{l}\text { Atención psicológica y social de niños y adolescentes hasta los 17 años } \\
\text { y su núcleo familiar. Producción de materiales educativos sobre la } \\
\text { cultura de la no violencia. }\end{array}$ \\
\hline Dirección & 3ra. Av. 11-28 zona 1 Edificio Guerra; Guatemala \\
\hline Teléfono & 2253-5303 \\
\hline Correo electrónico & conacmi@itelgua.com \\
\hline Cobertura & Guatemala, Jalapa, Escuintla, Baja Verapaz \\
\hline Contacto & Licdo. Miguel Angel López, Director \\
\hline
\end{tabular}

\begin{tabular}{|l|l|}
\hline Nombre institucional & Defensoría de la Mujer \\
\hline Misión & $\begin{array}{l}\text { Coordinadora Nacional de Asistencia Legal Gratuita a la víctima y sus } \\
\text { familiares. Violencia de todo tipo contra la mujer. }\end{array}$ \\
\hline Servicios & $\begin{array}{l}\text { Atención psicológica en crisis, sensibilización ante la violencia, acom- } \\
\text { pañamiento a la víctima durante el juicio. Procesos legales de filiación y } \\
\text { pensiones de alimentos. Atienden víctimas y sus hijos. Investigación so- } \\
\text { cial y seguimiento según necesidades sociales y familiares, visitas domi- } \\
\text { ciliarias. Protección de niños separados de la familia por orden judicial. }\end{array}$ \\
\hline Dirección & $\begin{array}{l}11 \text { calle. 9-38, zona 1 Guatemala } \\
\text { Teléfono }\end{array}$ \\
\hline 2232-9502 / 2253-3468 / 2253-3497; emergencia 1571 \\
\hline Correo electrónico & violenciaintrafamiliar@hotmail.com \\
\hline Cobertura & $\begin{array}{l}\text { Quetzaltenango, Jutiapa, Petén, Cobán, Villa Nueva, Mixco, Amatitlán, } \\
\text { Chiquimula, pero no todas las oficinas cuentan con todos los servicios }\end{array}$ \\
\hline Contacto & $\begin{array}{l}\text { Amalia Mazariegos - Corrdinadora; Alba Alburez - Jefe Unidad } \\
\text { Psico-Social }\end{array}$ \\
\hline
\end{tabular}




\section{Guatemala}

\begin{tabular}{|l|l|}
\hline Nombre institucional & Fundación Sobrevivientes \\
\hline Misión & $\begin{array}{l}\text { Contribuir a la prevención, sanción y erradicación de la violencia } \\
\text { contra las mujeres y la niñez en Guatemala. Acceso a la justicia por } \\
\text { parte de las mujeres y la niñez víctimas de violencia. }\end{array}$ \\
\hline Servicios & $\begin{array}{l}\text { Procesos legales penales y civiles, atención psicológica, asistencia } \\
\text { social y económica. Refugio temporal para mujeres sobrevivientes. }\end{array}$ \\
\hline Dirección & 12 Calle 11-63 zona 1, Guatemala \\
\hline Teléfono & 2245-30oo \\
\hline Correo electrónico & www.sobrevivientes.org, sobrevivientesfundacion@yahoo.es \\
\hline Cobertura & Nivel nacional \\
\hline Contacto & Vivian Dardón, Gestora de Proyectos; Berta Aída Batres, Psicóloga \\
\hline
\end{tabular}

\begin{tabular}{|l|l|}
\hline Nombre institucional & GGM - Grupo Guatemalteco de Mujeres \\
\hline Misión & $\begin{array}{l}\text { Políticas públicas en favor de la mujer y reducción de la violencia. } \\
\text { CAIMU: programa de apoyo y seguridad integral, atención directa a las } \\
\text { mujeres víctimas de violencia. Investigación, incidencia política, comu- } \\
\text { nicación, sensibilización y prevención de violencia contra la mujer. }\end{array}$ \\
\hline Servicios & $\begin{array}{l}\text { Atención integral: médica, psicológica, social y legal. Albergue: madres } \\
\text { con hijos, varones hasta los 13 años, capacidad para 40 personas. } \\
\text { Reciben adolescentes embarazadas por orden del juez. }\end{array}$ \\
\hline Dirección & 2a calle 8-13, zona 1 Guatemala \\
\hline Teléfono & 2230-2674 / 2250-O273 \\
\hline Correo electrónico & ggms@intelnet.net.gt, ggmprogramaapoyoyseguridad@gmail.com \\
\hline Cobertura & $\begin{array}{l}\text { Guatemala, Escuintla, Rabinal, Suchitepequez, todas con el mismo } \\
\text { modelo de atención integral }\end{array}$ \\
\hline Contacto & Aída Saravia, Coordinadora \\
\hline
\end{tabular}




\section{Guatemala}

\begin{tabular}{|l|l|}
\hline Nombre institucional & Médicos Sin Fronteras \\
\hline Misión & $\begin{array}{l}\text { Proyecto violencia sexual. Implementación y monitoreo de modelo de } \\
\text { atención médica, psicológica y social en diferentes clínicas y hospitales. }\end{array}$ \\
\hline Servicios & $\begin{array}{l}\text { Atención en crisis. Terapia psicológica. Seguimiento médico. } \\
\text { Reincersión social y protección de menores. }\end{array}$ \\
\hline Dirección & 12 calle, 22-75, zona 11, Residenciales San Jorge; Guatemala \\
\hline Teléfono & 2473-7405 / 2474-5830 \\
\hline Correo electrónico & $\begin{array}{l}\text { msfchsecre@itelgua.com; msfch-guate-hom@geneva.msf.org } \\
\text { www.guatemala.msf.mx }\end{array}$ \\
\hline Cobertura & Guatemala \\
\hline Contacto & Kassia Echavarri Queen, Coordinadora General \\
\hline
\end{tabular}

\begin{tabular}{|l|l|}
\hline Nombre institucional & Misión Internacional de Justicia \\
\hline Misión & $\begin{array}{l}\text { Organización sin fines de lucro defensora de los derechos de los niños } \\
\text { y jóvenes víctimas de violencia sexual. }\end{array}$ \\
\hline Servicios & $\begin{array}{l}\text { Servicios psicológicos y legales para víctimas de violencia sexual. } \\
\text { Preparación psicológica y emocional para enfrentar los procesos } \\
\text { legales. Terapia psicológica, apoyo médico y para la educación. } \\
\text { Apoyo con traslados de familias en riesgo. }\end{array}$ \\
\hline Dirección & 11 calle 3-38 zona 1 Guatemala \\
\hline Teléfono & 2463-5550 \\
\hline Correo electrónico & jortiz@ijm.org \\
\hline Cobertura & $\begin{array}{l}\text { Guatemala y sus municipios. Otros departamentos en casos } \\
\text { excepcionales }\end{array}$ \\
\hline Contacto & Licda. Jessica Ortiz, Directora de Casos y Operaciones \\
\hline
\end{tabular}




\section{QUETZALTENANGO}

\section{Quetzaltenango}

\begin{tabular}{|l|l|}
\hline Nombre institucional & Asociación Nuevos Horizontes \\
\hline Misión & $\begin{array}{l}\text { Reivindicar los derechos humanos de las mujeres, la niñez y adolescen- } \\
\text { cia, cambiando las relaciones desiguales de poder y discriminación. }\end{array}$ \\
\hline Servicios & $\begin{array}{l}\text { Atención integral a sobrevivientes de violencia intrafamiliar, violencia } \\
\text { sexual y contra las mujeres. Atención inicial, psicológica, social y } \\
\text { jurídica. Albergue para mujeres, madres con hijos menores de 13 años, } \\
\text { y adolescentes enviadas por juzgados. }\end{array}$ \\
\hline Dirección & 3a Calle D 3-55, Zona 1, Quetzaltenango \\
\hline Teléfono & 7765-0141 / 7761-4328 / 7823-1042 \\
\hline Correo electrónico & honoris17@yahoo.es www.ahnh.org \\
\hline Cobertura & Toda la República de Guatemala \\
\hline Contacto & Lilian Wug Maldonado, Coordinadora \\
\hline
\end{tabular}

\begin{tabular}{|l|l|}
\hline Nombre institucional & DEMI - Defensoría de la Mujer Indígena \\
\hline Misión & $\begin{array}{l}\text { Defender y promover el pleno ejercicio de los derechos de las mujeres } \\
\text { indígenas para contribuir a la erradicación de todas las formas de } \\
\text { violencia y discriminación contra la mujer indígena. }\end{array}$ \\
\hline Servicios & $\begin{array}{l}\text { Atención en idioma indígena y con pertinencia cultural. Asistencia } \\
\text { jurídica, social y psicológica. Procesos pensión alimenticia. }\end{array}$ \\
\hline Dirección & 14 ave. "A" 8-25 zona 3. Quetzaltenango \\
\hline Teléfono & $7723-0977$ / 7736-8997 \\
\hline Correo electrónico & demixela@yahoo.com; delegadaxela@yahoo.com \\
\hline Cobertura & Quetzaltenango y Totonicapán. \\
\hline Contacto & Natividad Batz, Delegada \\
\hline
\end{tabular}




\section{Quetzaltenango}

\begin{tabular}{|l|l|}
\hline Nombre institucional & $\begin{array}{l}\text { Defensa Pública - Coordinadora Nacional de Atención a la } \\
\text { Víctima }\end{array}$ \\
\hline Misión & $\begin{array}{l}\text { Brindar apoyo legal a mujeres y menores de escasos recursos, víctimas } \\
\text { de violencia. }\end{array}$ \\
\hline Servicios & $\begin{array}{l}\text { Procesos legales, estudios socio-económicos y visitas domiciliarias. } \\
\text { Evaluaciones psicológicas para juicios y atención en crisis. }\end{array}$ \\
\hline Dirección & Callejón 8 0-37 zona 7 Quetzaltenango \\
\hline Teléfono & $7736-8947 ;$ Emergencia: 5509-1561 cel: 50293223 \\
\hline Correo electrónico & No disponible \\
\hline Cobertura & Quetzaltenango y San Marcos \\
\hline Contacto & Licda. Fevy Magdely Irramajo Morales, Abogada \\
\hline
\end{tabular}

\begin{tabular}{|l|l|}
\hline Nombre institucional & CDH - Centro de Desarrollo Humano \\
\hline Misión & Apoyo a mujer víctima de violencia con escasos recursos económicos. \\
\hline Servicios & $\begin{array}{l}\text { Asistencia a mujeres que son víctimas de cualquier tipo de violencia } \\
\text { y con escasos recursos. Contactan abogados con precios mínimos. } \\
\text { Apoyo psicológico y social. }\end{array}$ \\
\hline Dirección & 6a. Calle diagonal 12-65 zona 1, Quetzaltenango \\
\hline Teléfono & 7761-2807 \\
\hline Correo electrónico & titaug@hotmail.com \\
\hline Cobertura & Quetzaltenango \\
\hline Contacto & Marta Urquizo, Directora \\
\hline
\end{tabular}




\section{SACATEPÉQUEZ}

\section{La Antigua Guatemala}

\begin{tabular}{|c|c|}
\hline Nombre institucional & APROFAM \\
\hline Misión & $\begin{array}{l}\text { Servicios de salud con énfasis en salud sexual y reproductiva, con } \\
\text { enfoque en el bienestar integral de las personas. }\end{array}$ \\
\hline Servicios & $\begin{array}{l}\text { Atención médica para hombres y mujeres. Programas de jóvenes, VIH, } \\
\text { embarazo en adolescentes. Orientación a víctimas de violencia sobre } \\
\text { los recursos legales. }\end{array}$ \\
\hline Dirección & 6 calle Poniente \# 46, La Antigua Guatemala \\
\hline Teléfono & $7832-2978$ \\
\hline Correo electrónico & Iruano@aprofam.org.gt \\
\hline Cobertura & Sacatepéquez \\
\hline Contacto & Dr. Luis Roberto Ruano \\
\hline
\end{tabular}

\begin{tabular}{|l|l|}
\hline Nombre institucional & Hospital Nacional \\
\hline Misión & $\begin{array}{l}\text { Brindar atención médica, psicológica y social a la persona que lo } \\
\text { necesite. }\end{array}$ \\
\hline Servicios & $\begin{array}{l}\text { Atención médica, psico-social, ya sea en consulta externa o } \\
\text { emergencia. Comité de violencia: orientación y capacitación a } \\
\text { víctimas y como trabajo de prevención. }\end{array}$ \\
\hline Dirección & Aldea San Felipe de Jesús, La Antigua Guatemala \\
\hline Teléfono & $7831-1399 / 25$ \\
\hline Correo electrónico & trabajosocialhmpb@gmail.com \\
\hline Cobertura & Sacatepéquez y otros departamentos \\
\hline Contacto & Licda. Brenda de Avalos, Trabajadora Social \\
\hline
\end{tabular}




\section{La Antigua Guatemala}

\begin{tabular}{|l|l|}
\hline Nombre institucional & Ministerio Público - Oficina de Atención a la Víctima \\
\hline Misión & $\begin{array}{l}\text { Atención víctimas directas e indirectas de delitos en contra de la vida, } \\
\text { integridad física, y delitos sexuales, entre otros. }\end{array}$ \\
\hline Servicios & $\begin{array}{l}\text { Atención en crisis, asistencia para iniciar procesos legales y medidas } \\
\text { de protección. Asistencia social y psicológica. }\end{array}$ \\
\hline Dirección & 1 calle del Chajon \#5, La Antigua Guatemala \\
\hline Teléfono & $4534-5467$ \\
\hline Correo electrónico & fdsacatepequez@mp.org.gt \\
\hline Cobertura & Todo el departamento \\
\hline Contacto & Licda Carmen Ma. Santos Pérez, Coordinadora \\
\hline
\end{tabular}

\begin{tabular}{|l|l|}
\hline Nombre institucional & PGN - Procuraduría General de la Nación \\
\hline Misión & $\begin{array}{l}\text { Representante de menores, ancianos y discapacitados en estado de } \\
\text { abandono y promover acciones judiciales y extrajudiciales para prote- } \\
\text { ger a la familia, haciendo énfasis en la mujer. }\end{array}$ \\
\hline Servicios & $\begin{array}{l}\text { Toma de denuncias por mandato de la ley y grupos de } \\
\text { autoayuda para mujeres víctimas de violencia. }\end{array}$ \\
\hline Dirección & 2da. Sur No. 11 Interior Convento Ruinas de Santa Clara \\
\hline Teléfono & 7832-9454 \\
\hline Correo electrónico & pgn.sacatepequez@hotmail.es \\
\hline Cobertura & Sacatepéquez \\
\hline Contacto & Licda. Vanesa Rodas, Delegada \\
\hline
\end{tabular}




\section{SOLOLÁ}

\section{Panajachel}

\begin{tabular}{|c|c|}
\hline Nombre institucional & COISOLA \\
\hline Misión & $\begin{array}{l}\text { Promover el acceso a la justicia a través de litigio estratégico en } \\
\text { casos de abuso sexual, pornografía infantil y violencia en contra de } \\
\text { la mujer. Incidencia política, reforzar la Red de Derivación y asentar } \\
\text { políticas públicas municipales. Reducir impunidad y aumentar penas } \\
\text { a violadores y abusadores. }\end{array}$ \\
\hline Servicios & $\begin{array}{l}\text { Modelo de atención integral a la víctima. Acompañamiento y peritaje } \\
\text { psicológico. Grupos de autoayuda en áreas rurales del departamento. } \\
\text { Evaluación social y psicológica de la víctima. }\end{array}$ \\
\hline Dirección & Calle Monterrey, Zona 2 (Pegado Hospedaje Sto Domingo) \\
\hline Teléfono & 4604-5517, fax: 7953-9101 \\
\hline Correo electrónico & ana-coisola@hotmail.com, pana_coisola@hotmail.com \\
\hline Cobertura & Municipios Sololá \\
\hline Contacto & $\begin{array}{l}\text { Neu Serena, Directora Programa Acceso a la Justicia; } \\
\text { Carmen Peneleu, Psicóloga }\end{array}$ \\
\hline
\end{tabular}




\section{Sololá}

\begin{tabular}{|l|l|}
\hline Nombre institucional & DEMI - Defensoría de la Mujer Indígena \\
\hline Misión & $\begin{array}{l}\text { Defender y promover el pleno ejercicio de los derechos de las mujeres } \\
\text { indígenas para contribuir a la erradicación de todas las formas de } \\
\text { violencia y discriminación contra la mujer indígena. }\end{array}$ \\
\hline Servicios & $\begin{array}{l}\text { Atención en idioma indígena y con pertinencia cultural. Asistencia } \\
\text { juridica, social y psicológica. Procesos pensión alimenticia. }\end{array}$ \\
\hline Dirección & 6 Av. y 13 Calle Zona 2, Sololá \\
\hline Teléfono & 2251-7415 \\
\hline Correo electrónico & demisolola@yahoo.com; delegadasolola@yahoo.com \\
\hline Cobertura & Sololá, Quetzaltenango, Alta Verapaz, Totonicapán \\
\hline Contacto & Dominga Vásquez, Delegada; Eliza Tereto, Abogada \\
\hline
\end{tabular}

\begin{tabular}{|l|l|}
\hline Nombre institucional & PDH - Procuraduría de los Derechos Humanos \\
\hline Misión & $\begin{array}{l}\text { Fiscalizadora de entes del estado, orientación legal de acuerdo al caso. } \\
\text { Acompañamiento como observadores en procesos legales. }\end{array}$ \\
\hline Servicios & $\begin{array}{l}\text { Reciben denuncias directas por parte de las víctimas, charlas sobre } \\
\text { prevención de violencia. }\end{array}$ \\
\hline Dirección & 5 Av. 8-20 Zona 1 \\
\hline Teléfono & $7762-3642 / 7721-7766$ \\
\hline Correo electrónico & solola_pdh@yahoo.com \\
\hline Cobertura & Departamento Sololá \\
\hline Contacto & Aura Ramos, Oficial de Defensoría \\
\hline
\end{tabular}




\section{TOTONICAPÁN}

\section{Totonicapán}

\begin{tabular}{|l|l|}
\hline Nombre institucional & Juzgado de Familia \\
\hline Misión & Recibir y tramitar denuncias. \\
\hline Servicios & $\begin{array}{l}\text { Reciben denuncias directas por parte de las víctimas. Dictámenes } \\
\text { psicológicos y estudios socio-económicos. Terapia de familia y pareja } \\
\text { en algunos casos. }\end{array}$ \\
\hline Dirección & 7a. Calle, zona 4, Totonicapán \\
\hline Teléfono & 7766-4047 \\
\hline Correo electrónico & No disponible \\
\hline Cobertura & Departamento Totonicapán \\
\hline Contacto & Dinora Ochoa, Psicóloga \\
\hline
\end{tabular}

\begin{tabular}{|l|l|}
\hline Nombre institucional & Oficina de Atención a la Víctima, Ministerio Público \\
\hline Misión & $\begin{array}{l}\text { Atención inmediata a víctimas de violencia. Diagnóstico victimológico, } \\
\text { orientación y seguimiento a casos. }\end{array}$ \\
\hline Servicios & $\begin{array}{l}\text { Estabilización en crisis. Evaluación emocional y psicológica, peritaje } \\
\text { psicológico. }\end{array}$ \\
\hline Dirección & 7ạ. Calle 8-40 Zona 3, Totonicapán \\
\hline Teléfono & $5733-3755$ \\
\hline Correo electrónico & cardambrosio@gmail.com \\
\hline Cobertura & Departamento Totonicapán \\
\hline Contacto & Aura Carolina Ambrosio, Psicóloga \\
\hline
\end{tabular}




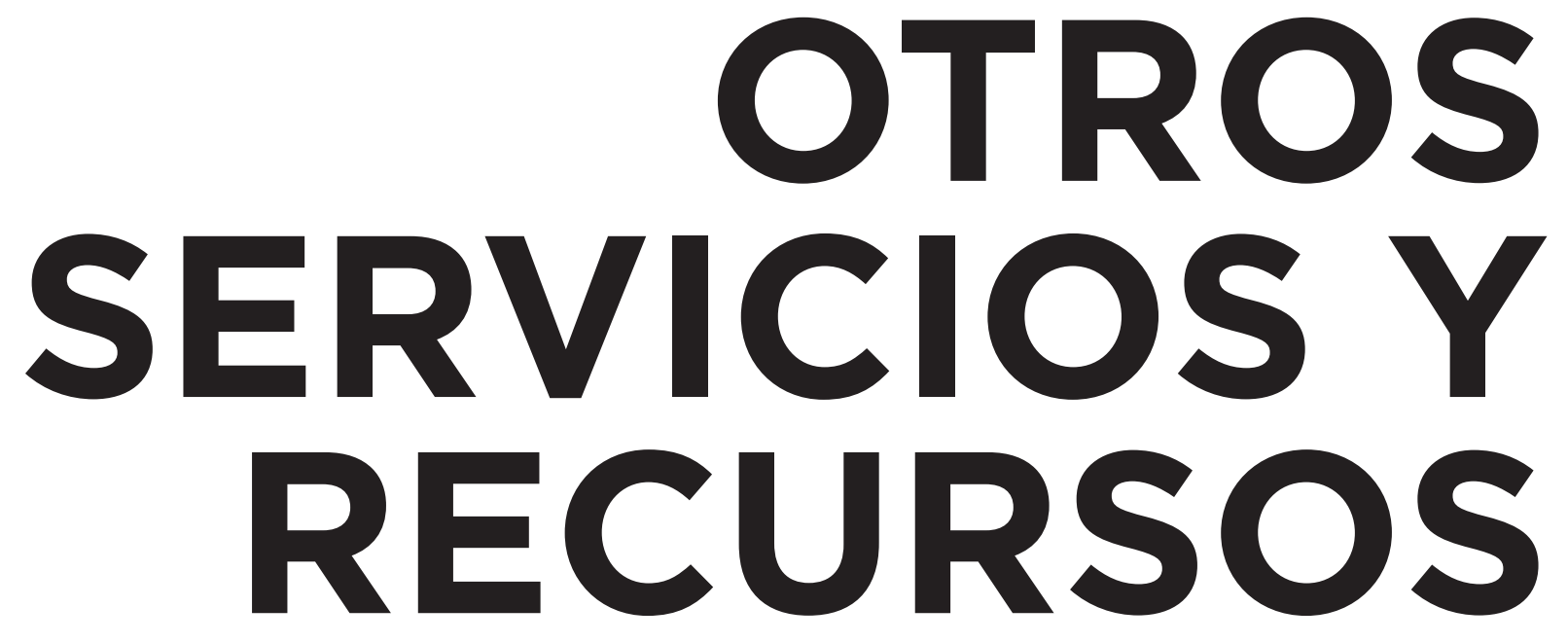





\section{ALTA VERAPAZ}

\section{Cobán}

\begin{tabular}{|l|l|}
\hline Nombre institucional & APROFAM \\
\hline Misión & $\begin{array}{l}\text { Servicios de salud para hombres, mujeres y niños con énfasis en } \\
\text { salud sexual y reproductiva y bienestar integral de las personas. }\end{array}$ \\
\hline Servicios & $\begin{array}{l}\text { Consulta interna y externa, control embarazo y atención de partos. } \\
\text { Programa salud sexual y reproductiva para jóvenes, VIH, embarazo } \\
\text { en adolescentes, consejería (psicología). APA - Atención post- } \\
\text { aborto. }\end{array}$ \\
\hline Dirección & Barrio San Bartolomé, 6 Av 3-51 Zona 1, Cobán Alta Verapaz \\
\hline Teléfono & 7951-1263, 1800 o05-6836 \\
\hline Correo electrónico & jhernandez@aprofam.org.gt \\
\hline Cobertura & Alta Verapaz \\
\hline Contacto & Licda. Juana Marta Hernández, Administradora \\
\hline
\end{tabular}

\begin{tabular}{|l|l|}
\hline Nombre institucional & ASECSA - Asociación de Servicios Comunitarios de Salud \\
\hline Misión & $\begin{array}{l}\text { Promoción de salud comunitaria en la población rural indígena y } \\
\text { ladina pobre que no tiene acceso a servicios de salud estatales o } \\
\text { privados. }\end{array}$ \\
\hline Servicios & $\begin{array}{l}\text { Desarrollo integral de la mujer, capacitación y talleres sobre } \\
\text { prevención de violencia. Acceso a medicamentos esenciales y } \\
\text { naturales. Escuela de formación política. }\end{array}$ \\
\hline Dirección & 19 Calle 3-13 Zona 10, Barrio La Libertad, Cobán Alta Verapaz \\
\hline Teléfono & 7941-7106 / 7952-1498; cel. 4586-4415 \\
\hline Correo electrónico & asecsanororiente@yahoo.es \\
\hline Cobertura & Cobán, Petén, Rabinal, Totonicapán y Chimaltenango \\
\hline Contacto & Liliana Laj, Encargada proyecto desarrollo político \\
\hline
\end{tabular}




\section{Cobán}

\begin{tabular}{|l|l|}
\hline Nombreinstitucional & Juzgado de Familia \\
\hline Misión & Recibir y tramitar denuncias. \\
\hline Servicios & $\begin{array}{l}\text { Trámites de divorcios, abandono de hogar, juicios por pensión de } \\
\text { alimentos. }\end{array}$ \\
\hline Dirección & 1 Calle 4-48 zona 3, Cobán Alta Verapaz. \\
\hline Teléfono & $7952-1510$ \\
\hline Correo electrónico & jdofamilia-av@hotmail.com \\
\hline Cobertura & Cabecera de Cobán, Alta Verapaz \\
\hline Contacto & Carlos Humberto Hom Caal, Secretario \\
\hline
\end{tabular}

\begin{tabular}{|c|c|}
\hline Nombre Institucional & $\begin{array}{l}\text { Juzgado Niñez y Adolescencia y Adolescentes en Conflicto } \\
\text { con la Ley Penal }\end{array}$ \\
\hline Misión & $\begin{array}{l}\text { Brindar protección a niños y adolescentes que sufren abusos o son } \\
\text { amenazados sus derechos humanos. }\end{array}$ \\
\hline Servicios & $\begin{array}{l}\text { Investigación de los casos de abuso o violencia en contra de } \\
\text { menores y otorgar medidas de protección. Evaluación psicológica } \\
\text { y social. Pedagogo y trabajadores sociales facilitan procesos de } \\
\text { reinserción social. }\end{array}$ \\
\hline Dirección & 5av. 1-64 Zona 4, Cobán Alta Verapaz \\
\hline Teléfono & $7952-9562$ \\
\hline Correo electrónico & No disponible \\
\hline Cobertura & Alta y Baja Verapaz \\
\hline Contacto & Mario Rene Xul Tun, Secretario de Juzgado \\
\hline
\end{tabular}




\section{Cobán}

\begin{tabular}{|l|l|}
\hline Nombre institucional & Oficina de Equidad de Género \\
\hline Misión & $\begin{array}{l}\text { Atención de casos de trata de personas, femicidios, violencia sexual } \\
\text { e intrafamiliar. }\end{array}$ \\
\hline Servicios & $\begin{array}{l}\text { Orientación ruta de la denuncia. Capacitación sobre temas de equidad } \\
\text { de género, VIH, tipos de violencia, femicidio y trata de personas. }\end{array}$ \\
\hline Dirección & 1ra. calle 5-12 zona 2 \\
\hline Teléfono & cel. 4349-7242 \\
\hline Correo electrónico & lijcareujo@yahoo.com \\
\hline Cobertura & San Pedro Carchá y Cobán Alta Verapaz \\
\hline Contacto & Agente Carmen Cacao, Coordinadora \\
\hline
\end{tabular}

\begin{tabular}{|l|l|}
\hline Nombre institucional & Pastoral de la Niñez en Desamparo \\
\hline Misión & $\begin{array}{l}\text { Propiciar apoyar y acompañar alternativas que contribuyan a un } \\
\text { desarrollo integral y autogestionario de la niñez y juventud más } \\
\text { desfavorecida de la región, promoviendo la organización y derechos } \\
\text { que les permitan nuevos y mejores espacios de vida. }\end{array}$ \\
\hline Servicios & $\begin{array}{l}\text { Apoyo a niños en nivel escolar para su educación, mejorar su } \\
\text { desarrollo integral, como también que conozcan sus derechos. } \\
\text { Recreación y deportes. Asesoría legal y acompañamiento a las } \\
\text { madres y menores en caso de violación de sus derechos y violencia } \\
\text { intrafamiliar. Escuela de padres. }\end{array}$ \\
\hline Dirección & 4a calle 8-40, zona 3, Cobán Alta Verapaz \\
\hline Teléfono & 4129-1698 \\
\hline Correo electrónico & pninez@gmail.com \\
\hline Cobertura & Comunidades y municipios de Alta Verapaz \\
\hline Contacto & Nélida Maldonado, Directora \\
\hline
\end{tabular}




\section{Tactic}

\begin{tabular}{|l|l|}
\hline Nombre institucional & OMM - Oficina Municipal de la Mujer \\
\hline Misión & $\begin{array}{l}\text { Institucionalizada en las estructura Municipal, incidiendo, impulsando, } \\
\text { elaborando y asesorando la formación de políticas públicas } \\
\text { municipales con equidad de género. }\end{array}$ \\
\hline Servicios & $\begin{array}{l}\text { Orientación y acompanamiento a víctimas de violencia, está } \\
\text { constituida por un equipo que contribuye a promover la equidad. } \\
\text { Ayudas con víveres, medicinas, gastos funerarios. Diplomado mensual } \\
\text { para mujeres en temas de participacion política, salud sexual y } \\
\text { reproductiva, violencia. }\end{array}$ \\
\hline Dirección & 2da Calle O7-13 Zona 1, Tactic, Alta Verapaz \\
\hline Teléfono & 4604-5517; fax: 7953-9101 \\
\hline Correo electrónico & carolina.gozalez78@hotmail.com \\
\hline Cobertura & Comunidades y cabecera municipal de Tactic \\
\hline Contacto & Nancy Catalina Molina, Coordinadora Interina \\
\hline
\end{tabular}




\section{CHIMALTENANGO}

\section{Chimaltenango}

\begin{tabular}{|l|l|}
\hline Nombre institucional & OMM - Oficina Municipal de la Mujer \\
\hline Misión & $\begin{array}{l}\text { Promover la equidad de género. OMM Institucionalizada en las } \\
\text { estructuras municipales, incidiendo, impulsando, elaborando y } \\
\text { asesorando la formación de políticas públicas en favor del desarrollo } \\
\text { de la mujer. }\end{array}$ \\
\hline Servicios & $\begin{array}{l}\text { Politicas públicas. Mujeres gestoras de su propio desarrollo. } \\
\text { Orientación y acompañamiento legal a mujeres víctimas de violencia. }\end{array}$ \\
\hline Dirección & $\begin{array}{l}\text { Distrito Noroccidente, Lote 1 Manzana F Col. Sta Teresita, } \\
\text { Chimaltenango }\end{array}$ \\
\hline Teléfono & 5525-7474 \\
\hline Correo electrónico & lorenitamontufa@gmail.com \\
\hline Cobertura & Todo el departamento de Chimaltenango \\
\hline Contacto & Lorena Montúfar, Asistencia Técnica. \\
\hline
\end{tabular}

\begin{tabular}{|l|l|}
\hline Nombre institucional & $\begin{array}{l}\text { Programa de Desarrollo Económico y Social de la Mujer Kichin } \\
\text { Konojel }\end{array}$ \\
\hline Misión & $\begin{array}{l}\text { Es una asociación de mujeres Maya que promueve y aplica aquellos } \\
\text { valores de justicia, igualdad de género, equidad, libertad y democracia. }\end{array}$ \\
\hline Servicios & $\begin{array}{l}\text { Proyectos de educación, socio-productivos, de capacitación } \\
\text { y participación cívico-política, artesanía, etc., con enfoque de } \\
\text { género y ecológico, de acuerdo a la cosmovisión maya. Asesoría } \\
\text { y acompañamiento a mujeres víctimas de violencia en casos } \\
\text { legales. Talleres con grupos y organizaciones de base en temas de } \\
\text { discriminacion étnica, interculturalidad y justicia. }\end{array}$ \\
\hline Dirección & \begin{tabular}{l}
9 Calle 5-17 Zona 1, Quintas 1, Chimaltenango \\
\hline Teléfono
\end{tabular} \\
\hline 7839-7033 / 7839-2419 \\
\hline Correo electrónico & konojel@hotmail.com \\
\hline Cobertura & $\begin{array}{l}7 \text { municipios en Chimaltenango (Acatenango, Parramos, Comalapa, Sta } \\
\text { Apolonia, San Martín Jilotepeque y Zaragoza) y Escuintla }\end{array}$ \\
\hline Contacto & Antonieta Castro, Directora; Licdo. Ruben Ortiz, Coordinador \\
\hline
\end{tabular}




\section{San Andrés Itzapa}

\begin{tabular}{|l|l|}
\hline Nombre institucional & $\begin{array}{l}\text { Fundación Integral de Desarrollo Sostenible y Medio } \\
\text { Ambiente (FIDESMA) }\end{array}$ \\
\hline Misión & $\begin{array}{l}\text { Programas definidos que abarquen el tema ambiental, el área } \\
\text { productiva, la salud preventiva, la educación y capacitación, la } \\
\text { atención a discapacitados y familias en riesgo. }\end{array}$ \\
\hline Servicios & $\begin{array}{l}\text { Programa Mujer Rural: micro préstamos y capacitaciones en } \\
\text { proyectos productivos y temas de la mujer. }\end{array}$ \\
\hline Dirección & Cantón San Cristóbal, San Andrés Itzapa \\
\hline Teléfono & $7849-5220$ \\
\hline Correo electrónico & fidesmaguate@yahoo.com \\
\hline Cobertura & Chimaltenango, Sacatepéquez y Sololá \\
\hline Contacto & Margarita Caté, Presidenta \\
\hline
\end{tabular}




\section{San Juan Comalapa}

\begin{tabular}{|l|l|}
\hline Nombre institucional & ASOINDEMA - Asociación Integral de Desarrollo Maya \\
\hline Misión & $\begin{array}{l}\text { Fomentar el desarrollo integral, social y ecónomico de las mujeres } \\
\text { socias. Promover la capacitación y participación de la mujer. }\end{array}$ \\
\hline Servicios & $\begin{array}{l}\text { Orientación y capacitación a mujeres sobre tema de violencia de } \\
\text { género. Promover capacitación y participación de las mujeres, apoyar } \\
\text { a las mujeres en actividades productivas en agricultura, pecuarias, } \\
\text { artesanías, etc. }\end{array}$ \\
\hline Dirección & 1a Calle A O-25 Zona 2, Comalapa \\
\hline Teléfono & 7849-8443 / 7849-8228 \\
\hline Correo electrónico & asoindema@intelnet.net.gt \\
\hline Cobertura & Área rural y urbana Comalapa \\
\hline Contacto & Rutilia Roquel, Directora \\
\hline
\end{tabular}

\begin{tabular}{|l|l|}
\hline Nombre institucional & Chuwi Tinamit \\
\hline Misión & $\begin{array}{l}\text { Programa salud para niños en áreas rurales. Enfermeros viajan a áreas } \\
\text { rurales para dar capacitación en temas de salud y atender niños con } \\
\text { desnutrición. }\end{array}$ \\
\hline Servicios & $\begin{array}{l}\text { Programa nutricional para niños. Educación en salud sexual y } \\
\text { reproductiva para jóvenes. }\end{array}$ \\
\hline Dirección & O Av A 1-22 Zona 4, San Juan Comalapa, Chimaltenango \\
\hline Teléfono & $7849-8181$ \\
\hline Correo electrónico & chuwi2439@gmail.com \\
\hline Cobertura & Área rural Comalapa, Chimaltenango \\
\hline Contacto & Licdo. Valeriano Pichiya, Director \\
\hline
\end{tabular}




\section{GUATEMALA}

\section{Guatemala}

\begin{tabular}{|l|l|}
\hline Nombre institucional & Asociación VIVA \\
\hline Misión & $\begin{array}{l}\text { Trabajar en favor de la niñez abusada, maltratada o abandonada. } \\
\text { Rescate de niños en la calle. }\end{array}$ \\
\hline Servicios & $\begin{array}{l}\text { Sistema de mejoramiento de calidad de hogares para niños. Apoyar } \\
\text { hogares legalmente constituidos a través de módulos de desarrollo } \\
\text { para el personal y apoyo para la implementación de modelos de } \\
\text { servicios integrales y de calidad. }\end{array}$ \\
\hline Dirección & 30 Avenida 6-20 Zona 11 Residenciales V, Guatemala \\
\hline Teléfono & 2439-6895 / 2439-6763 \\
\hline Correo electrónico & tiempoderescate@yahoo.com; hmoreno@redviva.org, \\
\hline Cobertura & Guatemala, Quiché, Verapaces, Parramos, Villa Nueva \\
\hline Contacto & Hugo Moreno, Director \\
\hline
\end{tabular}

\begin{tabular}{|l|l|}
\hline Nombre institucional & Tierra Viva \\
\hline Misión & $\begin{array}{l}\text { Organización feminista. Promover derechos sexuales y reproductivos } \\
\text { de la mujer. Enfoque político: mujer como ciudadana politica, con } \\
\text { derecho a tomar decisiones. Avanzar y mantener avances legislativos } \\
\text { en favor de la mujer. }\end{array}$ \\
\hline Servicios & $\begin{array}{l}\text { Acompañamiento para hacer denuncias ante las autoridades } \\
\text { correspondientes sobre violencia en contra de la mujer, especialmente } \\
\text { abuso sexual. Formación y capacitación a jovenes en instituciones } \\
\text { educativas, grupos de mujeres líderes en comunidades y comadronas. }\end{array}$ \\
\hline Dirección & 3ra. Av 10-18 Zona 1, Guatemala \\
\hline Teléfono & 2251-7372 / 2253-7244 \\
\hline Correo electrónico & tierraviva@guate.net.gt; moralesevelyn22@yahoo.com \\
\hline Cobertura & $\begin{array}{l}\text { 6 departamentos en Guatemala: Ciudad de Guatemala, San Marcos, } \\
\text { Sololá, Chimaltenango, Chiquimula }\end{array}$ \\
\hline Contacto & Evelyn Morales, Coordinadora \\
\hline
\end{tabular}




\section{QUETZALTENANGO}

\section{Quetzaltenango}

\begin{tabular}{|l|l|}
\hline Nombre institucional & AMOIXQUIK - Asociación de Mujeres de Occidente \\
\hline Misión & $\begin{array}{l}\text { Procesos de formación y sanación personal. Empoderamiento } \\
\text { humano, étnico cultural, económico y político. Avanzar en el } \\
\text { cumplimento pleno de sus derechos colectivos y específicos como } \\
\text { mujeres, sustentándose en la creación y recreación de valores, } \\
\text { pensamientos, actitudes, prácticas y propuestas que les permita } \\
\text { alcanzar su desarrollo integral, con equidad de género. }\end{array}$ \\
\hline Servicios & $\begin{array}{l}\text { Acompañamiento y grupos de autoayuda a la mujer víctima de } \\
\text { violencia. Sensibilización en contra de la violencia. }\end{array}$ \\
\hline Dirección & Diagonal 12 8-69 zona 1 Quetzaltenango \\
\hline Teléfono & 7761-O397 \\
\hline Correo electrónico & demujeres@itelgua.com \\
\hline Cobertura & San Marcos, Quetzaletenango, Totonicapán y Sololá \\
\hline Contacto & Ana Victoria García, Subdirectora. \\
\hline
\end{tabular}

\begin{tabular}{|c|c|}
\hline Nombre institucional & AMUTED - Asociación Mujer Tejedora del Desarrollo \\
\hline Misión & $\begin{array}{l}\text { Organización de mujeres que trabaja en pro del desarrollo integral } \\
\text { y empoderamiento de las mujeres indígenas, no indígenas, adultas } \\
\text { y jóvenes del área rural y urbana, promoviendo el autocuidado y el } \\
\text { desarrollo productivo y económico. }\end{array}$ \\
\hline Servicios & $\begin{array}{l}\text { Cursos productivos. Desarrollar proyectos comunitarios con mujeres. } \\
\text { Educación y sensibilización en temas de derechos y no violencia. } \\
\text { Orientación y acompañamiento en casos de violencia. }\end{array}$ \\
\hline Dirección & 9a. Calle C2O-42 A zona 1, Quetzaltenango \\
\hline Teléfono & 5515-9822; fax: 7765-5622 \\
\hline Correo electrónico & amuted@amuted.info \\
\hline Cobertura & 5 municipios del departamento \\
\hline Contacto & $\begin{array}{l}\text { Julia Sum, Coordinadora General; Francisca López, Técnica del } \\
\text { Proyecto Violencia Intrafamiliar }\end{array}$ \\
\hline
\end{tabular}




\section{Quetzaltenango}

\begin{tabular}{|l|l|}
\hline Nombre institucional & CEIPA - Centro Ecuménico de Integración Pastoral \\
\hline Misión & $\begin{array}{l}\text { Apoyo a niños y niñas trabajadores para que puedan continuar con su } \\
\text { educación y aprender sobre derechos humanos, entre otros temas. }\end{array}$ \\
\hline Servicios & $\begin{array}{l}\text { Educación primaria acelerada. Promoción de los derechos de la niñez } \\
\text { e incidencia para la formulación de las políticas públicas. Reciben } \\
\text { jóvenes embarazadas para que continúen sus estudios. }\end{array}$ \\
\hline Dirección & 20 Av. D1-75, zona 3 Residenciales la Democracia Quetzaltenango \\
\hline Teléfono & $7767-3853$ \\
\hline Correo electrónico & ssljuventudkiche@hotmail.com \\
\hline Cobertura & 11 municipios de Quetzaltenango y 8 en Retalhuleu. \\
\hline Contacto & Héctor de León, Coordinador del Programa de Educación \\
\hline
\end{tabular}

\begin{tabular}{|l|l|}
\hline Nombre Institucional & Concepto 10 Paz y Desarrollo \\
\hline Misión & $\begin{array}{l}\text { Prestar servicios legales a mujeres víctimas de violencia y con escasos } \\
\text { recursos. }\end{array}$ \\
\hline Servicios & $\begin{array}{l}\text { Atender a mujeres con escasos de recursos para servicios y asesoría } \\
\text { legal. }\end{array}$ \\
\hline Dirección & 16 Avenida 3-17 zona 1, Quetzalenango \\
\hline Teléfono & $7763-0457$ \\
\hline Correo Electrónico & tomyolvia2000@yahoo.com \\
\hline Cobertura & Quetzaltenango \\
\hline Contacto & Tomy Oliva de López, Directora \\
\hline
\end{tabular}




\section{Quetzaltenango}

\begin{tabular}{|l|l|}
\hline Nombre institucional & Cooperación Alemana \\
\hline Misión & Brindar servicios médicos a personas de escasos recursos. \\
\hline Servicios & Brindar atención médica y psicológica, incluidas víctimas de violencia. \\
\hline Dirección & 3ra. calle 3-58, zona 3, Quetzaltenango \\
\hline Teléfono & 7761-5320 \\
\hline Correo electrónico & www.ascagp.com.org \\
\hline Cobertura & Quetzaltenango \\
\hline Contacto & Ingrid Xicara, Administradora \\
\hline
\end{tabular}

\begin{tabular}{|l|l|}
\hline Nombre institucional & Parlamento Guatemaltenco de la Niñez y Adolescencia - IDEI \\
\hline Misión & $\begin{array}{l}\text { Que la niñez y la adolescencia Maya, Garífuna, Xincas y Mestizos } \\
\text { desarrollen protagonismo y liderazgo a nivel municipal, regional y } \\
\text { nacional para el fortalecimiento de la paz en Guatemala. }\end{array}$ \\
\hline Servicios & $\begin{array}{l}\text { Prevención y educación en salud sexual y reproductiva, derechos } \\
\text { humanos e incidencia política para niños y jóvenes. Fortalecer la salud } \\
\text { de la mujer indígena, promoción de sus derechos, e incidencia para } \\
\text { erradicar prácticas dañinas. }\end{array}$ \\
\hline Dirección & 7 calle 4-24 zona 1, Quetzaltenango \\
\hline Teléfono & 7761-9419 \\
\hline Correo electrónico & $\begin{array}{l}\text { www.asociacionidei.org, parlamentoniñezguat@gmail.com, josem. } \\
\text { yac@gmail.com }\end{array}$ \\
\hline Cobertura & Quetzaltenango \\
\hline Contacto & José Yac, Coordinador y Ana Cristina Benavente \\
\hline
\end{tabular}




\section{Quetzaltenango}

\begin{tabular}{|c|c|}
\hline Nombre institucional & Primeros Pasos \\
\hline Misión & $\begin{array}{l}\text { Mejorar la calidad de vida de las poblaciones en el área rural que } \\
\text { carecen de acceso a servicios médicos. Brindar educación preventiva } \\
\text { a través de programas educativos de salud y facilitar el acceso a } \\
\text { servicios médicos y educación. }\end{array}$ \\
\hline Servicios & $\begin{array}{l}\text { Desarrollar proyectos comunitarios para mejorar la salud. Charlas con } \\
\text { mujeres de carácter preventivo en diferentes temas de salud. }\end{array}$ \\
\hline Dirección & $\begin{array}{l}\text { 6a. Calle 7-31, zona } 1 \text { Quetzaltenango, Cantón Tierra Colorada Baja, } \\
\text { Sector I, Quetzaltenango, Guatemala }\end{array}$ \\
\hline Teléfono & $7968-5777 / 4393-6848$ \\
\hline Correo electrónico & info@primerospasos.org; www.primerospasos.org \\
\hline Cobertura & 10 comunidades rurales de Quetzaltenango \\
\hline Contacto & Margarita Tay, Encargada Clínica \\
\hline
\end{tabular}

\begin{tabular}{|l|l|}
\hline Nombre institucional & Project Hope \\
\hline Misión & $\begin{array}{l}\text { Combinar microcreditos con programas de educación en salud para } \\
\text { que las mujeres puedan emprender algún negocio y alcanzar su } \\
\text { desarrollo. }\end{array}$ \\
\hline Servicios & $\begin{array}{l}\text { Prevención a través de educación a mujeres socias del programa de } \\
\text { créditos sobre violencia, auto-estima, ruta de la denuncia, marco legal. } \\
\text { Asesoría y créditos para micro-empresas. }\end{array}$ \\
\hline Dirección & 34 Av 9-50 Zona 3 Resideciales DELCO \\
\hline Teléfono & 7767-6931 / 7765-8287 \\
\hline Correo electrónico & www.projecthope.org \\
\hline Cobertura & Area rural y urbana de Quetzaltenango \\
\hline Contacto & Brenda Yes, Coordinadora Salud \\
\hline
\end{tabular}




\section{SACATEPÉQUEZ}

\section{La Antigua Guatemala}

\begin{tabular}{|l|l|}
\hline Nombre institucional & Área de Salud \\
\hline Misión & $\begin{array}{l}\text { Coordinación con centros de salud y hospitales para atender a } \\
\text { la mujer víctima de violencia, darle asesoría y acompañamiento. } \\
\text { Seguimiento de casos en el departamento. }\end{array}$ \\
\hline Servicios & $\begin{array}{l}\text { Programa de jóvenes “Por un Mundo Feliz." Realizan talleres sobre } \\
\text { violencia, salud sexual y reproductiva y ecología. }\end{array}$ \\
\hline Dirección & 7 Ave Norte No 53, La Antigua Guatemala \\
\hline Teléfono & 5679-1395 \\
\hline Correo electrónico & karina_carranzam@hotmail.com \\
\hline Cobertura & Sacatepéquez \\
\hline Contacto & Karina Carranza, Coordinadora Programa de Violencia \\
\hline
\end{tabular}

\begin{tabular}{|l|l|}
\hline Nombre institucional & $\begin{array}{l}\text { Centro de Documentación e Información para Adolescentes - } \\
\text { Centro de Salud Departamental }\end{array}$ \\
\hline Misión & $\begin{array}{l}\text { Educación para adolescentes en salud sexual y reproductiva, alcohol } \\
\text { y tabaquismo. }\end{array}$ \\
\hline Servicios & $\begin{array}{l}\text { Charlas sobre educación sexual, noviazgo, círculo de la violencia. } \\
\text { Se realizan a través de las escuelas pero cualquier joven puede } \\
\text { participar. }\end{array}$ \\
\hline Dirección & Carretera a San Felipe de Jesús \\
\hline Teléfono & 7832-0483 \\
\hline Correo electrónico & cediasac@gmail.com \\
\hline Cobertura & Antigua, Sumpango, San Lucas, Santiago Sacatepéquez e Izabal \\
\hline Contacto & Karina Carranza, Coordinadora Programa de Violencia \\
\hline
\end{tabular}




\section{SOLOLÁ}

\section{Panajachel}

\begin{tabular}{|l|l|}
\hline Nombre institucional & $\begin{array}{l}\text { Asociación Comunitaria para el Desarrollo Sololateco - } \\
\text { Xocomil }\end{array}$ \\
\hline Misión & Promover la educación en salud y salud comunitaria. \\
\hline Servicios & $\begin{array}{l}\text { Prevención y educación en salud sexual y reproductiva y reducción de } \\
\text { muerte materno-infantil. }\end{array}$ \\
\hline Dirección & Calle El Chali 4-12 Zona 2, Panajachel \\
\hline Teléfono & $7762-0770 ;$ cel. 5704-6789 \\
\hline Correo electrónico & xocomil-asoc@hotmail.com \\
\hline Cobertura & 5 municipios del departamento \\
\hline Contacto & Moisés Paulino Cutz Toc, Presentante Legal \\
\hline
\end{tabular}

\begin{tabular}{|l|l|}
\hline Nombre institucional & Redes Juveniles \\
\hline Misión & $\begin{array}{l}\text { Grupo organizado de adolescentes y jóvenes capacitándose en la } \\
\text { prevención del abuso y explotación sexual. Aumentar la participación } \\
\text { de la juventud en la sociedad civil. Fomentar políticas públicas en } \\
\text { favor de la niñez y la juventud. }\end{array}$ \\
\hline Servicios & $\begin{array}{l}\text { Formación de adolescentes en autoestima, protagonismo, reducción y } \\
\text { protección en contra la violencia. }\end{array}$ \\
\hline Dirección & Calle Principal Oo-O28 Zona 2, Panajachel \\
\hline Teléfono & 7762-2869 \\
\hline Correo electrónico & panajachel@pami-guatemala.org \\
\hline Cobertura & Panajachel \\
\hline Contacto & Hilario Us, Coordinador \\
\hline
\end{tabular}




\section{Santa Lucía Utatlán}

\begin{tabular}{|l|l|}
\hline Nombre institucional & Centro de Salud \\
\hline Misión & Proveer servicios médicos. \\
\hline Servicios & $\begin{array}{l}\text { Atender casos de violencia en contra de la mujer desde el punto de } \\
\text { vista médico. Tienen disponibilidad de anticonceptivos. }\end{array}$ \\
\hline Dirección & Santa Lucía Utatlán \\
\hline Teléfono & $7722-1466$ \\
\hline Correo electrónico & centrodesaludslu@yahoo.com \\
\hline Cobertura & Todo el departamento \\
\hline Contacto & Dr. Diego Hernández, Director \\
\hline
\end{tabular}

\begin{tabular}{|l|l|}
\hline Nombre institucional & Juzgado de Paz \\
\hline Misión & $\begin{array}{l}\text { Atender casos de delitos y hechos de violencia de todo tipo. Llevar a } \\
\text { cabo denuncias y procesos legales. }\end{array}$ \\
\hline Servicios & Servicios legales a mujeres víctimas de violencia. \\
\hline Dirección & Santa Lucía Utatlán \\
\hline Teléfono & $7722-1477$ \\
\hline Correo electrónico & No disponible \\
\hline Cobertura & Todo el departamento \\
\hline Contacto & Perfecto Xajil Martín, Juez \\
\hline
\end{tabular}




\section{Santiago Atitlán}

\begin{tabular}{|l|l|}
\hline Nombre institucional & $\begin{array}{l}\text { ADECCAP - Asociación de Desarrollo Comunitario del Cantón } \\
\text { Panajab }\end{array}$ \\
\hline Misión & $\begin{array}{l}\text { Organización local que trabaja en proyectos ambientales y en el } \\
\text { manejo de desechos sólidos. }\end{array}$ \\
\hline Servicios & $\begin{array}{l}\text { Trabajar con comunidad y sectores más vulnerables, generando } \\
\text { autogestión y continuidad de los proyectos. }\end{array}$ \\
\hline Dirección & Cantón Pachichaj, Santiago Atitlán \\
\hline Teléfono & $7721-7306$ \\
\hline Correo electrónico & adeccapanabaj@yahoo.com \\
\hline Cobertura & Santiago Atitlán, Panabaj \\
\hline Contacto & Francisco Coche, Director \\
\hline
\end{tabular}

\begin{tabular}{|l|l|}
\hline Nombre institucional & Asociación de Salud y Desarrollo Rixiin Tinamet \\
\hline Misión & $\begin{array}{l}\text { Organización de base comunitaria de origen maya que provee } \\
\text { servicios de salud enfocados en la atención materno-infantil y } \\
\text { salud sexual y reproductiva. Promover desarrollo integral de las } \\
\text { comunidades. }\end{array}$ \\
\hline Servicios & $\begin{array}{l}\text { Servicios de salud, consulta externa, laboratorio y exámenes médicos. } \\
\text { Formación y organización comunitaria, promover red de lideresas } \\
\text { comunitarias para la promoción de salud y educación en salud sexual } \\
\text { y reproductiva. Empoderamiento de la mujer. }\end{array}$ \\
\hline Dirección & Cantón Xechivoy, Santiago Atitlán \\
\hline Teléfono & 7721-7246 / 7721-7245 \\
\hline Correo electrónico & rxiintnamet@gmail.com; www.asociacionrxiintnamet.com \\
\hline Cobertura & Santiago Atitlán y San Juan La Laguna \\
\hline Contacto & Leticia Toj de Méndez, Directora \\
\hline
\end{tabular}




\section{Santiago Atitlán}

\begin{tabular}{|l|l|}
\hline Nombre institucional & Comite Pro-Seguridad y Desarrollo \\
\hline Misión & Reducir criminalidad y mantener la seguridad de la población. \\
\hline Servicios & $\begin{array}{l}\text { Trabaja por la seguridad del municipio en alianza con la oficina de } \\
\text { atención a la víctima. Acompañamiento a víctimas de violencia } \\
\text { intrafamiliar, violencia sexual y delitos en contra de menores. }\end{array}$ \\
\hline Dirección & Municipalidad, Santiago Atitlán \\
\hline Teléfono & $4772-5262 / 5999-2428$ \\
\hline Correo electrónico & No disponible \\
\hline Cobertura & Santiago y San Juan \\
\hline Contacto & Rafael Estrada, Procesos Jurídicos \\
\hline
\end{tabular}




\section{Sololá}

\begin{tabular}{|l|l|}
\hline Nombre institucional & Medicus Mundi Aragón \\
\hline Misión & $\begin{array}{l}\text { Fortalecimiento de la red de servicios de salud sexual y reproductiva } \\
\text { y salud materno-infantil. }\end{array}$ \\
\hline Servicios & $\begin{array}{l}\text { Talleres a mujeres en comunidades sobre temas de salud sexual } \\
\text { y reproductiva. Educación y manejo de emergencias neo-natales. } \\
\text { Materiales didácticos sobre SSR y derechos sexuales y reproductivos. }\end{array}$ \\
\hline Dirección & Edificio Municipalidad, Sololá \\
\hline Teléfono & No disponible \\
\hline Correo electrónico & mmasolola@gmail.com \\
\hline Cobertura & Nivel departamental de Sololá \\
\hline Contacto & $\begin{array}{l}\text { Mercedes Franco del Pozo, Coordinadora; Flor Barrera, Tec. Salud } \\
\text { Sexual }\end{array}$ \\
\hline
\end{tabular}

\begin{tabular}{|l|l|}
\hline Nombre Institucional & Programa Nacional de Resarcimiento \\
\hline Misión & $\begin{array}{l}\text { Atender a mujeres víctimas sobrevientes del conflicto armado en } \\
\text { Sololá y Chichicastenango. }\end{array}$ \\
\hline Servicios & Asesoría legal, acompañamiento psicosocial y grupos de autoayuda. \\
\hline Dirección & 5 Av. 14-28 Zona 1, Barrio El Carmen, Sololá \\
\hline Teléfono & $7762-3068$ \\
\hline Correo Electrónico & No disponible \\
\hline Cobertura & Sololá y Chichicastenango \\
\hline Contacto & Neida Porón, Coordinadora; Lucía Chávez, Psicóloga \\
\hline
\end{tabular}




\section{TOTONICAPÁN}

\section{Totonicapán}

\begin{tabular}{|l|l|}
\hline Nombre institucional & ACPDC - Asociación Pro-desarrollo Comunitario \\
\hline Misión & $\begin{array}{l}\text { Proyecto de salud sexual y reproductiva y distribución de medicina en } \\
\text { áreas rurales del departamento. }\end{array}$ \\
\hline Servicios & $\begin{array}{l}\text { Capacitación a jóvenes sobre diferentes temas como la prevención de } \\
\text { violencia. }\end{array}$ \\
\hline Dirección & Canton Xantún, Paraje Pujacar, Totonicapán \\
\hline Teléfono & $7766-3061$ / 5863-2591 \\
\hline Correo electrónico & acpdc@intelnet.net.gt y acpdc@turbonet.com \\
\hline Cobertura & Totonicapán y municipios aledaños \\
\hline Contacto & Victoriano Jesús Morales López, Representante Legal \\
\hline
\end{tabular}

\begin{tabular}{|l|l|}
\hline Nombre institucional & Área de Salud \\
\hline Misión & $\begin{array}{l}\text { Programa de salud reproductiva incluye ejes temáticos de violencia. } \\
\text { Capacitación a educadores programa "Vivir Bien". }\end{array}$ \\
\hline Servicios & $\begin{array}{l}\text { Educación y prevención en diferentes temas de salud sexual y } \\
\text { reproductiva. Refieren casos de violencia en contra de la mujer al } \\
\text { juzgado de familia. }\end{array}$ \\
\hline Dirección & 10 Avenida 1-31, Zona 2, Totonicapán \\
\hline Teléfono & $7766-2419$ \\
\hline Correo electrónico & merliga@hotmail.es \\
\hline Cobertura & Departamento de Totonicapán \\
\hline Contacto & Mercedes García Vásquez, Encargada Salud Sexual y Reproductiva \\
\hline
\end{tabular}




\section{Totonicapán}

\begin{tabular}{|l|l|}
\hline Nombre institucional & PDH - Procuraduría de Derechos Humanos \\
\hline Misión & $\begin{array}{l}\text { Fiscalizadora de entes del estado, orientación legal de acuerdo al } \\
\text { caso. Acompañamiento como observadores en casos legales. }\end{array}$ \\
\hline Servicios & $\begin{array}{l}\text { Documentar las denuncias, seguimiento a expedientes, medidas de } \\
\text { seguridad y seguimiento a los casos. }\end{array}$ \\
\hline Dirección & 3a calle y 16 Ave. Esquina, zona 2, Totonicapán \\
\hline Teléfono & $7766-1412$ / 7766-1081 \\
\hline Correo electrónico & auxtoto@gmail.com \\
\hline Cobertura & Departamento de Totonicapán \\
\hline Contacto & $\begin{array}{l}\text { Karen Irene Linares Robles, Auxiliar Departamental; } \\
\text { Flor Gómez, Oficial Analista }\end{array}$ \\
\hline
\end{tabular}




\section{ALBERGUES}

\section{Guatemala (San Cristóbal)}

\begin{tabular}{|l|l|}
\hline Nombre institucional & Buckner Orphan Care Guatemala \\
\hline Misión & Hogar para niños enviados por orden de juez. \\
\hline Servicios & Trabajo social y psicologia \\
\hline Dirección & Manzana 6, lote 15, sector B3 San Cristóbal zona 8 \\
\hline Teléfono & $2480-7345$ \\
\hline Correo electrónico & mabelbocigua@gmail.com \\
\hline Cobertura & Guatemala y sus municipios \\
\hline Contacto & Mabel de Jacome, Directora de trabajado Social \\
\hline
\end{tabular}

\section{La Antigua Guatemala}

\begin{tabular}{|l|l|}
\hline Nombre institucional & Asociación Nuestros Ahijados \\
\hline Misión & $\begin{array}{l}\text { Cuidar y educar a niños huérfanos, abandonados y a familias atadas a } \\
\text { la pobreza. }\end{array}$ \\
\hline Servicios & $\begin{array}{l}\text { Asistencia legal gratuita en casos de violencia en contra de menores y } \\
\text { mujeres. Servicios psicológicos, club de madres (autoayuda y motivan } \\
\text { asistencia donando verduras todos los viernes). Colegio pre-primaria, } \\
\text { primaria y básicos. Apadrinamiento de niños. Centro nutricional, } \\
\text { clínica médica y dental. Programa de construcción de vivienda. }\end{array}$ \\
\hline Dirección & Carretera San Felipe de Jesús No. 6, La Antigua Guatemala \\
\hline Teléfono & 5681-O182 \\
\hline Correo electrónico & david.arresis@anaguatemala.org \\
\hline Cobertura & Sacatepéquez y otros departamentos \\
\hline Contacto & Licdo. Jeremy David Arresis, Director \\
\hline
\end{tabular}




\section{Patzún}

\begin{tabular}{|l|l|}
\hline Nombre institucional & Hogar nuestros Pequeños Hermanos \\
\hline Misión & Hogar para niños, centro educativo pre-primaria, hospitalito. \\
\hline Servicios & Brindar educación a niños menores de 5 años. \\
\hline Dirección & 3Av \#6-10 Zona 1, Patzún \\
\hline Teléfono & 7839-8195 \\
\hline Correo electrónico & cnhfranciscanaspatzun@gmail.com \\
\hline Cobertura & Chimaltenango \\
\hline Contacto & Hna Ana Emilia Aguilar \\
\hline
\end{tabular}

\section{Quetzaltenango}

\begin{tabular}{|l|l|}
\hline Nombre Institucional & Hogar Temporal Quetzalteco \\
\hline Misión & Brindar atención y protección a niñas y niños \\
\hline Servicios & Servicio psicológico, legal, social y médico a niños del albergue. \\
\hline Dirección & 4ta. Ave 4-23, zona 5, Colonia Molina, Quetzaltenango \\
\hline Teléfono & 7767-5557 \\
\hline Correo electrónico & elibetyescobar@hotmail.com \\
\hline Cobertura & Quetzaltenango \\
\hline Contacto & Elibety Escobar Morales, Directora \\
\hline
\end{tabular}




\section{Santiago}

\begin{tabular}{|l|l|}
\hline Nombre institucional & Manos de Compasión \\
\hline Misión & Albergue Infantil \\
\hline Servicios & $\begin{array}{l}\text { Dar hogar, alimentación, vestido y educación a niños abandonados o } \\
\text { víctimas de violencia. }\end{array}$ \\
\hline Dirección & Lote 1-26 Bahía de Santiago, Aldea Tzanchaj \\
\hline Teléfono & 4407-0519 / 4407-1480 \\
\hline Correo electrónico & Manos Compasion@gmail.com, www.guatemalaministry.com \\
\hline Cobertura & Santiago Atitlán y municipios aledaños \\
\hline Contacto & David y Deborah Richard, Directores \\
\hline
\end{tabular}


